\title{
Effect of surface properties on momentum transfer to targets impacted by high- velocity sand slugs
}

\author{
A. Goel ${ }^{\mathrm{a}}$, T. Uth ${ }^{\mathrm{a}}$, H.N.G. Wadley ${ }^{\mathrm{b}}$ and V.S. Deshpande ${ }^{\mathrm{a}^{*}}$
}

\begin{abstract}
The response of dry and water saturated sand slugs impacting normally oriented and inclined rigid-stationary targets with four different surface coatings is measured with an emphasis on the quantification of the momentum transmitted from the slugs into the targets. The targets were coated with Alumina, PTFE, Aluminium or sand-paper layers in order to investigate the effect of varying surface hardness and surface roughness. In all the cases, the fraction of the slug momentum transferred into the target was equal for dry and water saturated sand slugs and also independent of the slug velocity over the range that is investigated here. For normal impacts, the surface coatings had no measurable influence on the momentum transfer into the targets and this was attributed to the symmetry of the impact event. However, the break of symmetry in the inclined impact cases resulted in two non-zero components of the net transmitted momentum into the targets and a strong influence of the surface coatings. This is attributed to friction between the sand particles and the target surface with the resultant transmitted momentum increasing in the order Alumina to PTFE to Aluminium to sand-paper surface coatings. In all cases, the transmitted momentum was less than the corresponding value under normal impact. Coupled discrete particle/ Lagrangian simulations of these experiments with the sand particles modelled as spheres captured the normal impact measurements with a high degree of fidelity. However, the simulations underestimated the transmitted momentum for the inclined impacts especially for the rough surface coatings such as the sand-paper: increasing the friction coefficient between the particles and the target in the simulations did not improve the predictions. We demonstrate that this discrepancy is due to the spherical particle assumption: in the experiments the sand particles are sub-spherical and this reduces the tendency of particles to roll on the target surface and thereby increases frictional interactions. Increasing the radius of gyration of particles decreased the discrepancy between the measurements and the predictions but yet could not accurately predict all components of the transmitted momentum. Most numerical calculations tend to use spherical particles to represent the impacting granular media. However, this study demonstrates the need to appropriately parameterise particle shape in such discrete particle calculations to accurately capture the granular media/ structure interactions.
\end{abstract}


Keywords: Granular material; Particle impact; Discrete particle simulation; Fluidstructure interaction; surface friction.

* Corresponding author. E-mail address: vsd@eng.cam.ac.uk 


\section{Introduction}

The design of vehicle underbody structures that can survive the impact of soil ejected by shallow-buried explosives has been a topic of considerable interest for many years. Numerous strategies have been proposed to improve the impulsive load resistance of these underbody structures including (i) replacing the monolithic underbody by sandwich panels [1-4], (ii) increasing the stand-off distance of the vehicle floor from the ground [1,5-7], and (iii) inclining the underbody with respect to the ground by making use of a V-shaped hull design [8-11] as sketched in Fig. 1. In all approaches, understanding the level of momentum transferred from the high-velocity soil ejecta onto the loaded structure (the target) is of primary importance to their successful implementation.

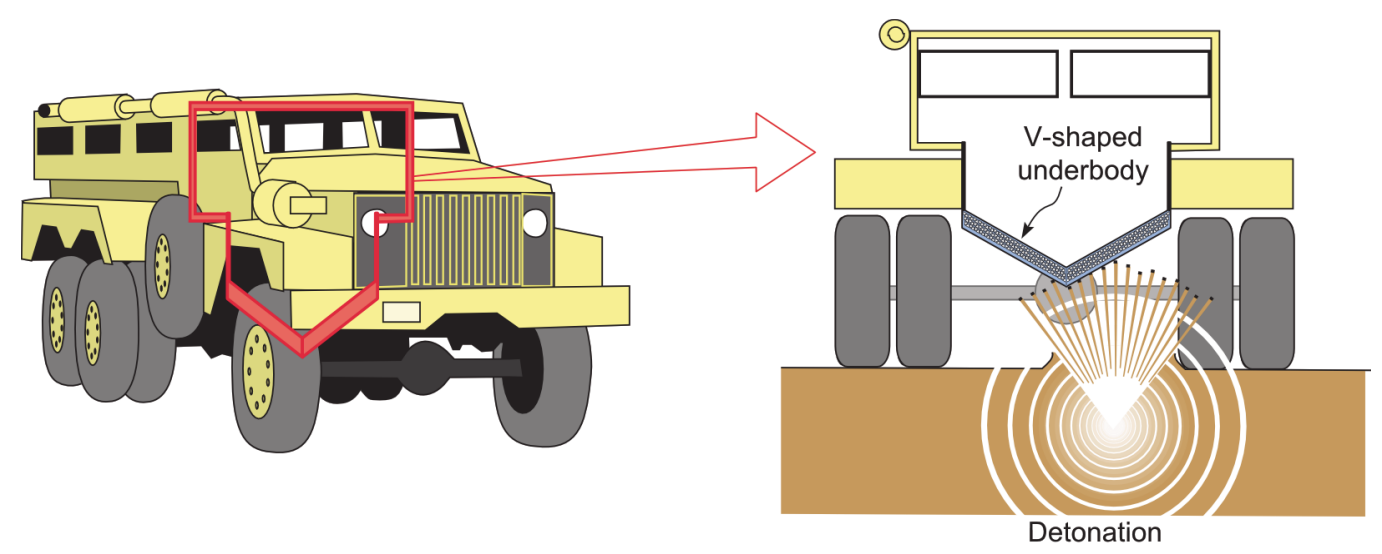

Figure 1: Schematic illustration of a vehicle with V-shaped underbody design to protect against the soil ejecta generated by the detonation of a shallow-buried explosive.

The phenomena that contribute to the dynamic loading of a structure following detonation of shallow-buried explosives are very complex. Empirical models that predict the impulsive loads imposed by soil ejecta [12] as well as structural design codes such as the one proposed by Morris [13] have helped inform more recent experimental characterizations of buried explosive events [14,15]. Moreover, experimental $[9,16]$ and recent numerical $[5,17]$ studies have confirmed that the soil impact is responsible for a substantial fraction of the blast load applied to a target structure, thus justifying the use of design codes such as those in [13] that neglect loading due to the accompanying air blast.

Experimental studies have shown that increasing the stand-off distance between the target and the explosive/ground also decreases the deflections of monolithic plates $[5,7,18]$ and sandwich panels [1]. This decrease has been attributed to a reduction in the momentum transfer from the ejected soil and detonation products to the target due to the spherical expansion of the ejecta; see experiments of Hlady [6] for rigid targets and Pickering et al. [7] for deformable plates. This reaffirms the importance of understanding the momentum transmitted into the targets from the soil ejecta.

Many numerical approaches have been proposed to model the complex dynamic interaction of granular sprays with structures. For example, Rimoli et al. [3] used the 
soil constitutive model of Deshpande et al. [19] to deduce the impulse applied to structures by explosively driven spherical sand shells, and then simulated the ensuing deformation of Aluminium monolithic and sandwich plates using a finite element method. Grujicic et al. [20-22] and Wang et al. [23] used coupled Eulerian/ Lagrangian simulations of landmine explosions, and compared their predictions with blast impulse and plate deformation measurements from Bergeron and Tremblay [9] and Foedinger [24]. More recently, coupled discrete particle/continuum simulations have been increasingly used to investigate the response of structures impacted by high-velocity granular media. For example, Borvik et al. [5] followed by Dharmasena et al. [1] and Holloman et al. [25,26] used this approach to simulate the response of a variety of monolithic and sandwich structures loaded by high-velocity sand sprays generated by explosive detonations. The importance of coupling the interaction of the granular medium with the dynamically changing geometry of the impacted surface was demonstrated by Wadley et al. [4] during a reinterpretation of the experiments reported by Rimoli et al. [3]. All these discrete particle approaches typically use spheres to represent the granular particles though some differences exist in individual implementations. For example, Liu et al. [2] and Dharmasena et al. [1] allow for three rotational and three translational degrees of freedom for the particles while the approaches of Borvik et al. [5] and Holloman et al. [25,26] restrict rotational motion of the spheres even though tangential frictional forces that result in torques on the particles are included in the analyses. It remains as yet unclear whether these differing assumptions affect the predictions.

There is considerable evidence that V-hull construction (Fig. 1) significantly enhances the survivability of vehicles subjected to impulsive loading from buried explosions. However, there is a paucity of data in the open literature, with most such studies restricted to rigid targets. If the ejecta launched by a buried explosion is brought to rest by a flat rigid target whose surface is perpendicular to the direction of particle impact, the full normal component of its momentum is transferred to the plate and the plate is subjected to the stagnation pressure associated with bringing a fluid to rest $[19,27]$. Experiments $[8,11]$ suggest that the momentum transferred from the ejecta into an inclined rigid target is less than that transferred into a normally oriented target while Benedetti [28] and Follett et al. [10] have reported similar findings for V-hulls made from Aluminium sheets and composite materials, respectively. Uth et al. [29] reported detailed laboratory scale measurements of all the components of the momentum transmitted into inclined monolithic and sandwich beams impacted by granular slugs comprising tungsten carbide particles. These detailed measurements indicated that while the resultant momentum transmitted into the inclined targets was indeed less than that for the normally oriented counterparts, it was significantly higher than that anticipated from a "water jet" like impact analysis [30,31] which neglects friction between the granular particles and the target. We note that the symmetry of the impact situation for normally oriented targets implies that friction does not affect the resultant transmitted momentum. However, friction is expected to influence the momentum transfer when symmetry of the impact geometry is broken. 
The symmetric impact of ejecta against a target as analysed in most model situations reported in the literature (see for example $[1,3-5,25,26]$ ) is expected to be a special case rather than the norm for practical situations. Thus, the problem of a granular slug impact against an inclined target, as investigated by Uth et al. [29], serves as a useful prototypical problem to both investigate the response of V-hull type structures and understand the general situation of a non-symmetric granular impact against a target. This is the primary focus of this study where we investigate the influence of surface type and inclination on the momentum transfer into rigid-stationary targets. Coupled discrete particle/Lagrangian numerical simulations that help identify the key physics governing the impact process are also reported to support interpretation of the experimental investigations.

\section{Experimental protocol}

Cylindrical slugs comprising silica sand particles were impacted against rigid stationary targets to measure the transmitted momentum. Fig. 2a depicts the experimental setup, which comprises four components (from right to left); (i) a gas gun to fire a solid projectile, which then accelerates the piston of (ii) a slug launcher apparatus based upon that developed by Park et al. [27]; (iii) a sand slug that initially rests inside the cylindrical cavity of the launcher; and (iv) the rigid stationary target, mounted on force sensors to measure the transmitted momentum. We proceed to briefly describe the four main components of the experimental setup sketched in Fig. 2a. 
(a)

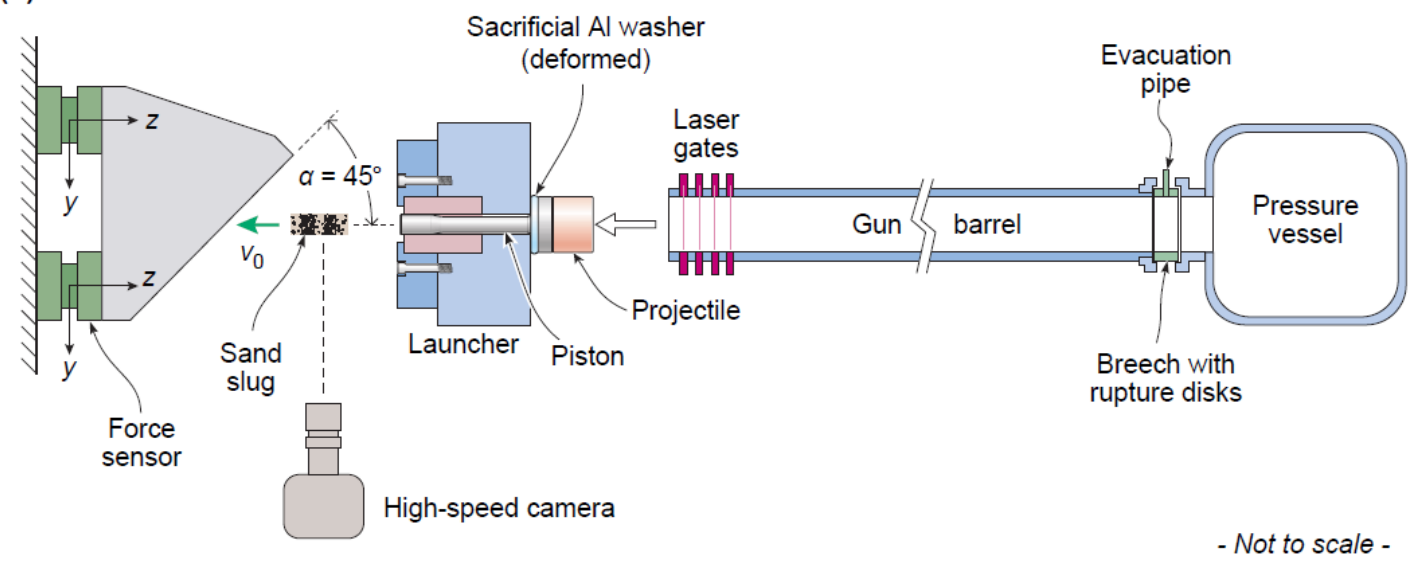

All dimensions in $\mathrm{mm}$

(b)

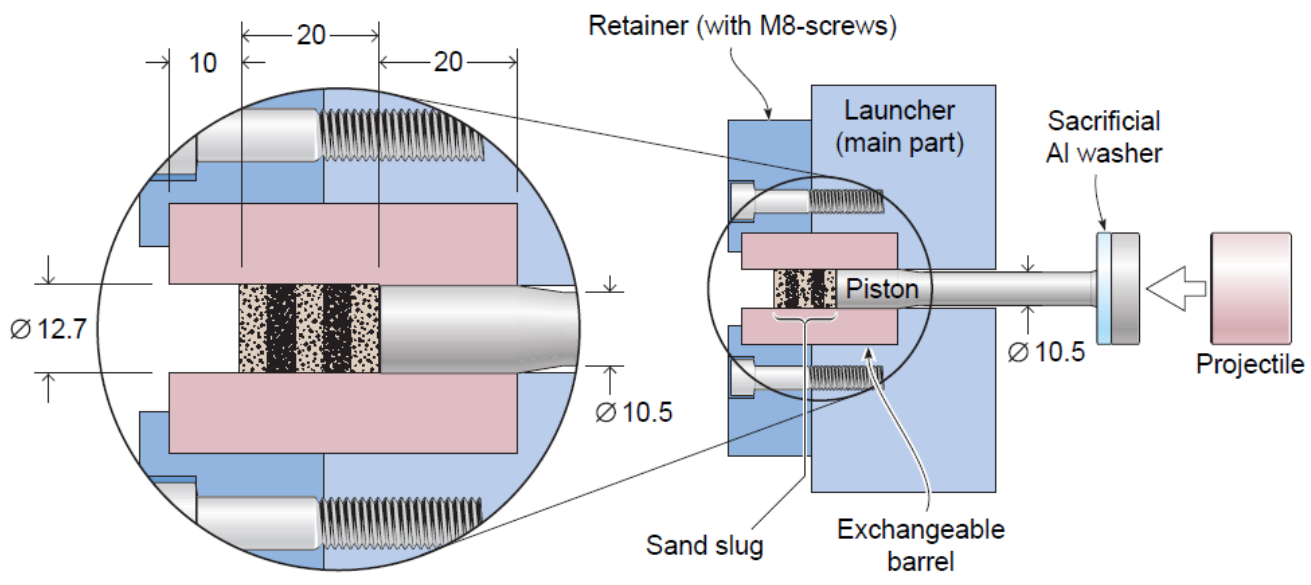

Figure 2: (a) Sketch of the overall setup used to investigate the normal and inclined impact of sand slugs against rigid-stationary targets. The setup includes a gas gun to fire the projectile, a launcher to generate the slug, and a rigid-stationary target. The impact event is imaged using a high-speed camera and the projectile velocity is measured using laser gates. (b) A sketch showing a cross-sectional view of the slug launcher with the inset showing a detailed view of the piston in contact with the slug. All dimensions are in $\mathrm{mm}$.

\subsection{Slug launcher}

The launcher was developed by Park et al. [27] and later modified by Uth and Deshpande [32] and Uth et al. [29]. A cross-sectional view of the slug launcher is sketched in Fig. $2 \mathrm{~b}$ and unless otherwise mentioned, all the components were made with low-carbon steel. It comprised a cylindrical cavity for the sand slug and a piston to push the slug out of the cavity when a projectile, fired from a gas gun, impacted the piston head. The launcher was bolted to a rigid support frame so that it remained stationary during the impact event. The launcher consisted of a thick-walled cylinder with an exchangeable barrel of inner diameter $12.7 \mathrm{~mm}$ and length $50 \mathrm{~mm}$. The piston had three distinct segments: a front, middle and back with dimensions as sketched in Fig. 2b: this segmented geometry was required to allow for both a tight fit of the piston front in contact with the sand and also to allow for "fattening" of the piston near the impacted end without jamming of the piston. The piston head at the back acts as an end-stop to arrest the piston. In addition, a $5 \mathrm{~mm}$ thick Aluminium alloy washer of inner diameter $12.7 \mathrm{~mm}$ and outer diameter $25 \mathrm{~mm}$ (equal to that of the piston 
head) was slid onto the piston until it was snug against the piston head. This washer cushioned the impact of the piston head against the launcher. A retainer was bolted to the front of the main part of the launcher to hold the exchangeable barrel in place. Before the launch process, the slug sat inside the exchangeable barrel such that there was a $10 \mathrm{~mm}$ gap between the front of the slug and the end of the exchangeable barrel: trial-and-error showed that this extra cavity length helped to maintain the shape of the launched slug. Furthermore, the sand particles abrade the inner wall of the exchangeable inner barrel and hence it was replaced after every three tests to ensure a good sliding fit between the piston and the barrel.

\subsection{Silica sand slugs}

Two types of sand slugs were employed: (i) dry sand slugs of mass and (ii) water saturated slugs of mass $5.14 \mathrm{~g}$ with $4.2 \mathrm{~g}$ of sand and $0.94 \mathrm{~g}$ of water filling all interstitial spaces between the silica particles. The silica sand ${ }^{1}$ particles were subspherical with particle sizes in the range 150-300 $\mu \mathrm{m}$ : see micrograph in Fig. 3. Before the launch, the cylindrical slugs had a diameter equal to that of the launcher and length. The slugs were prepared as detailed in Park et al. [27] and comprised 5 equal width alternating layers of coloured and uncoloured particles so as to give the slug a zebra-striped appearance (Fig. 2b). This zebra pattern improved the visualisation of the deformation of the slug during free-flight.

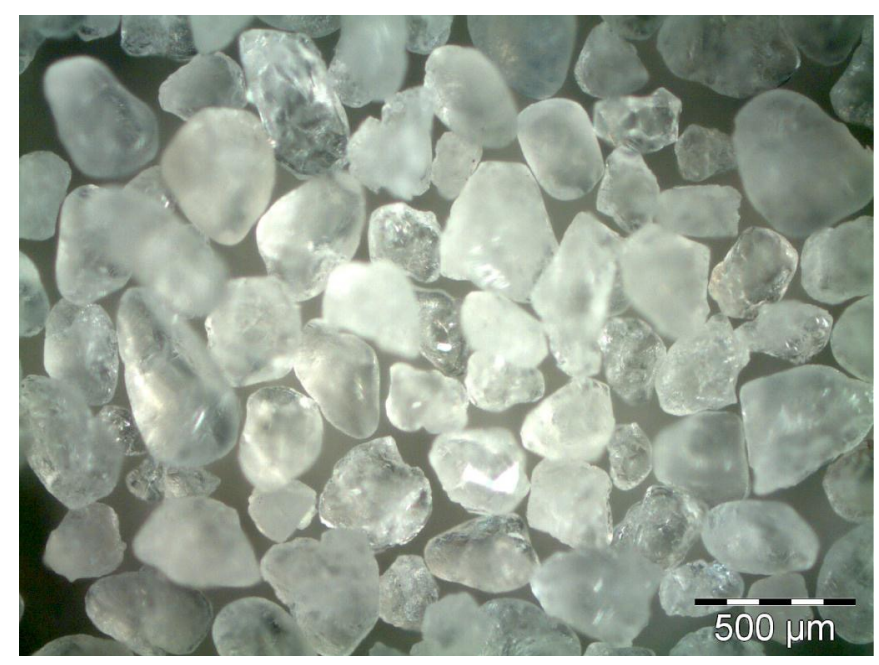

Figure 3: Optical micrograph of the sand particles used in both the dry and water saturated sand slugs.

\subsection{Gas gun to fire projectile}

A steel projectile of $105 \mathrm{~g}$ mass and $28.4 \mathrm{~mm}$ diameter was accelerated using a gas gun with a barrel length of $4.5 \mathrm{~m}$ and inner diameter of $28.5 \mathrm{~mm}$, as shown in Fig. 2a. No sabot was employed and the breach mechanism of the gun was formed by bursting copper diaphragms. The impact velocities of the projectile against the piston of the launcher ranged from to ; the velocity of the projectile was measured at the exit of the

\footnotetext{
${ }^{1}$ BS 1881-131:1998, fraction D - David Ball Group, Wellington Way, Bourn, CB23 2TQ, UK
} 
barrel using laser gates. While higher velocities would have been desirable in order to replicate granular media velocities in landmine explosions, impact of the piston at higher velocities irreparably damages the launcher and this limits the maximum projectile velocities employed in this study to about. The impacted end of the piston was placed about $25 \mathrm{~mm}$ in front of the end of the gun barrel. The flight of the sand slug and the profile views of the spreading of the sand slug over the target were visualized using a Phantom v1610 digital camera ${ }^{2}$ operating at an inter-frame rate of with an exposure time of .

A magnified sketch of the impact of the projectile against the piston that pushes the slug out of the launcher is shown in Fig. 4a. Upon impact of the projectile, both elastic and (slower) plastic waves emanate from the impacted end and propagate towards the end of the piston in contact with the granular slug. These waves deform the piston and thus the end of the piston in contact with the slug does not attain its final velocity instantaneously. To illustrate this effect, we plot the displacement in Fig. $4 \mathrm{~b}$ of the four marker lines on the piston shown in Fig. 4a for a projectile impact speed. These displacements are plotted as a function of time, where corresponds to the instant of impact of the projectile. The displacement rates of the markers (i.e. the marker velocities) all reach a constant and equal value at large as seen in Fig. $4 \mathrm{~b}$. This is consistent with the fact that the deformation of the piston ceases sometime after the impact of the projectile, and the piston thereafter behaves as a rigid body. This temporally and spatially constant marker velocity is defined as the piston velocity. The piston velocities for all the experiments reported here were experimentally obtained in this manner, and the relation between and obtained via this procedure is shown in Fig. 4c. We shall refer to experiments via their piston velocity and this measurement will be directly used as an input to the simulations reported in Section 5, thereby avoiding the need to explicitly model the impact of the projectile against the piston.

${ }^{2}$ Vision Research, Priory Business Park, Stannard Way, Bedford, MK44 3RZ, UK. 
(a)
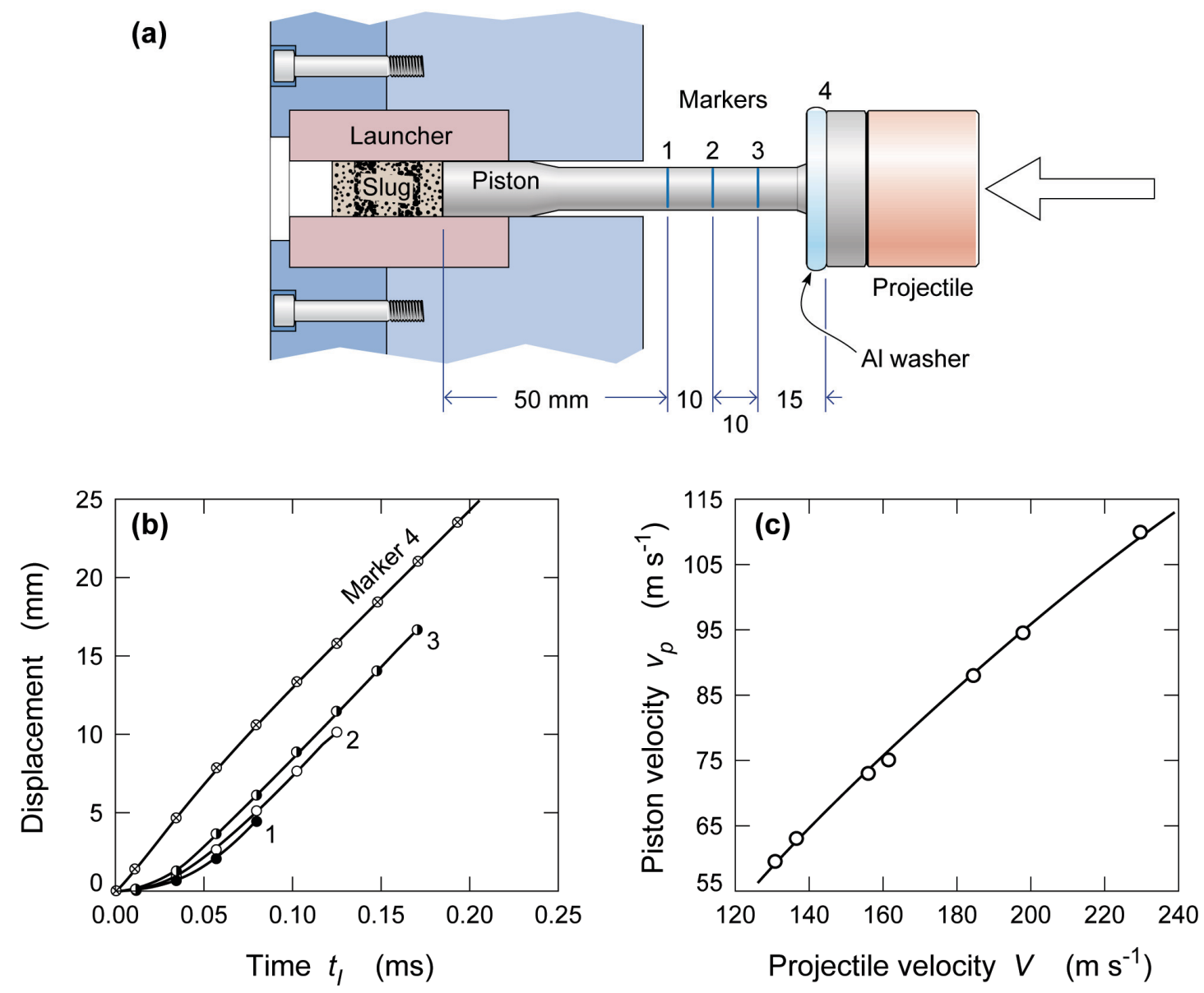

Figure 4: (a) Magnified view of the launcher with the piston that has just been impacted by the projectile. The locations of four marker lines on the piston are shown: the displacements of these markers are followed via the high-speed photography. (b) The temporal evolution of the displacements of the four marker lines in (a) for a projectile impact velocity. Here time corresponds to the instant of impact of the projectile. (c) The relation between the piston velocity and projectile velocity inferred from the high-speed photographs of the piston motion.

\section{$2.4 \quad$ Target design}

Experiments were conducted with rigid and stationary targets in one of two orientations as sketched in Fig. 5. In the "normal orientation" (Fig. 5a), the targets were oriented such that the slugs impacted at an angle to the face of the target while in the "inclined orientation", the slugs impacted at (Fig. 5b). The targets were $50 \mathrm{~mm}$ wide solid blocks of Aluminium with a mass of $1 \mathrm{~kg}$. The length of the target surface facing the slug impact was $50 \mathrm{~mm}$ for normal impact (i.e. a square target of dimensions ) and $100 \mathrm{~mm}$ for inclined impact case, as sketched in Fig. 5. This solid Aluminium target can be considered rigid for the levels of slug impact velocities and momenta considered here. 
The targets were in turn supported on two 3-component piezoelectric force sensors (Kistler ${ }^{3}$, type 9347C) that were used to measure the momentum transferred by the impacting slug into the target. These sensors measure forces in the three orthogonal directions, where the -axis was always defined in the direction of the incoming slug as shown in Fig. 5. The sensors could measure forces of up to $30 \mathrm{kN}$ in the -direction and $10 \mathrm{kN}$ in the - and -directions. The output from the sensors was conditioned using a Kistler 5001 charge amplifier and recorded with a digital oscilloscope (Tektronix ${ }^{4}$, TDS 3014B).
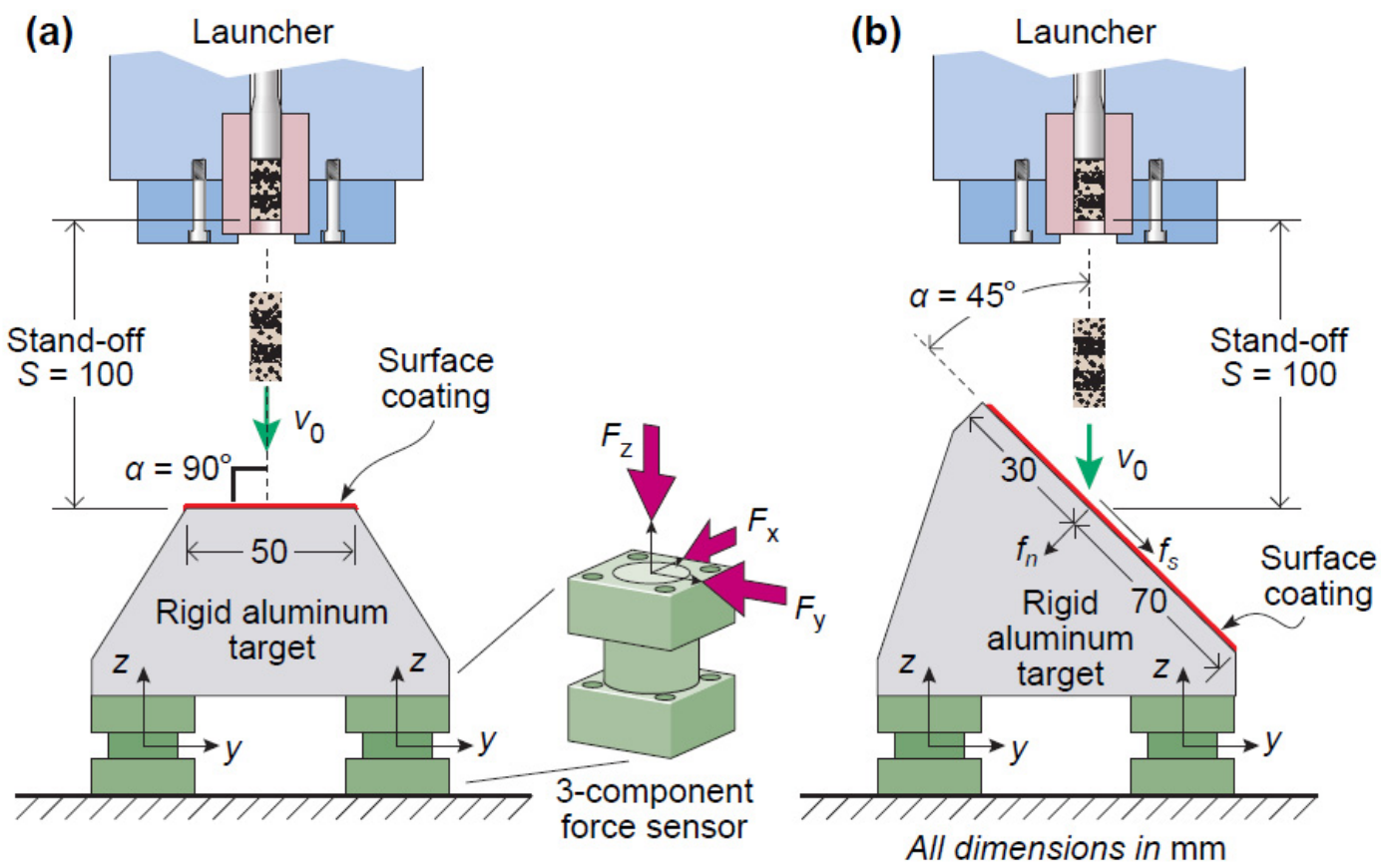

Figure 5: Sketches of the rigid stationary targets in the (a) normal and (b) inclined orientations. The coordinate system fixed to the support structure is sketched along with the piezoelectric force sensors used to measure the momentum transferred into the target. The inset in part (a) shows a detailed view of the force sensors and the three components of the measured forces. In both (a) and (b) we show a portion of the slug launcher in order to define the stand-off and the location of the slug impact on the target. All dimensions are in $\mathrm{mm}$.

A key objective of the experimental program was to determine the effects of the impacted surface properties (hardness and roughness) on the interaction of the sand slug with the target. For this purpose, 4 surface coatings with contrasting surface roughness and hardnesses were adhesively attached the surface of the rigid Aluminium target:

(i) $0.6 \mathrm{~mm}$ thick plate of solid Alumina (Aluminium Oxide - );

(ii) Sand-paper (grade 3M 251D P60-SC1) comprising a so-called "X-weight" cloth which is a heavy duty tear-resistant blend of cotton and polyester coated with Alumina particles having a grit size of $\mathrm{P} 60$;

\footnotetext{
${ }^{3}$ Kistler Instruments Ltd., Hook, Hampshire RG27 9GR, UK.

${ }^{4}$ Tektronix, P.O. Box 500, Beaverton, OR 97077, USA.
} 
(iii) $1.5 \mathrm{~mm}$ thick plate of the Aluminium alloy (grade EN AW-1050A H14); and

(iv) $1.5 \mathrm{~mm}$ thick plate of PTFE.

The mean roughness and Vickers hardness of the four coating layers are summarised in Table 1. We note that the hardness of the sand-paper is that measured by performing a Vickers indentation test on the sand-paper placed on a rigid foundation and represents a combination of the hardness of the backing cloth and the hardness of the Alumina grit (the hardness of the Alumina comprising the grit is ).

Table 1: The mean surface roughness and Vickers hardness of the four surface coatings employed in this study.

\begin{tabular}{|l|c|c|}
\hline & Mean roughness $(\mu \mathrm{m})$ & Vickers Hardness () \\
\hline Alumina & 0.69 & 1600 \\
\hline Sand-paper & 30.6 & 27.6 \\
\hline Aluminium & 0.15 & 39.2 \\
\hline PTFE & 1.06 & 3.7 \\
\hline
\end{tabular}

The target was positioned at a stand-off in all experiments, where is defined in Fig. 5 as the distance between the front of the stationary sand slug and the front face of the target at the centre of impact location.

\subsection{Temporal evolution and characterisation of slugs}

Sand slugs were generated by firing projectiles against the launcher piston which in turn generated piston velocities in the range - . In line with observations made for silica sand slugs [27,32] and Tungsten Carbide (WC) particle slugs [29], the velocity of the particles within the slugs remained temporally invariant after the slugs fully exit the launcher (i.e. the velocity of the slug was constant over the period during which observations could be made). As in previous studies, the particles within the slug acquired nearly no radial velocity but their axial velocity varied with position along the axis of the slug. To indicate this, Fig. 6a shows a montage of high-speed photographs showing the evolution (stretching) of the dry sand slug generated by impact with a piston speed. Note the time corresponds to the instant of the impact of the projectile against the piston. These images clearly show that the slug is elongating, consistent with sand particles having an axial velocity gradient along the slug with those at the front travelling more rapidly than at the tail.

In order to quantify the spatial gradient of the axial velocity, images from the highspeed camera were used to measure the velocities of the boundaries between the coloured and uncoloured sand particle layers. Measurements of the temporally invariant boundary velocities, where the superscript refers to the boundary number are plotted in Fig. $6 \mathrm{~b}$ for two selected values of (the boundaries are labelled in the 
inset of Fig. 6b). They clearly show the axial gradient in the particle velocities within the slug with the front of the slug having a higher velocity compared to the rear resulting in elongation of the slug during free-flight. Noting that each of the 5 layers of the slug has equal mass, the free-field momentum of the slug is

and the average slug velocity is then defined as. Subsequently, we shall refer to the velocity of slug via this average velocity .

(a)

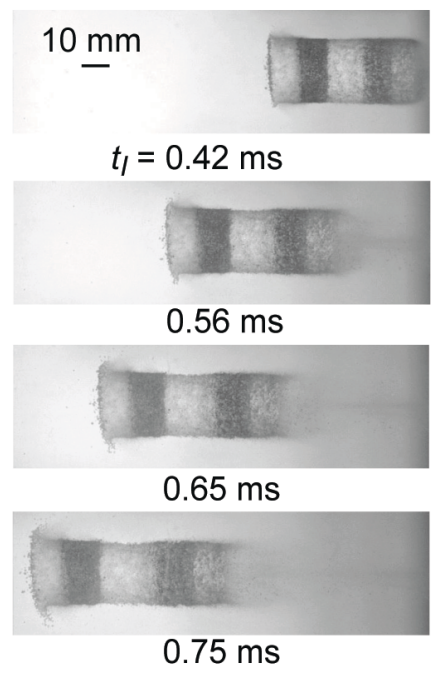

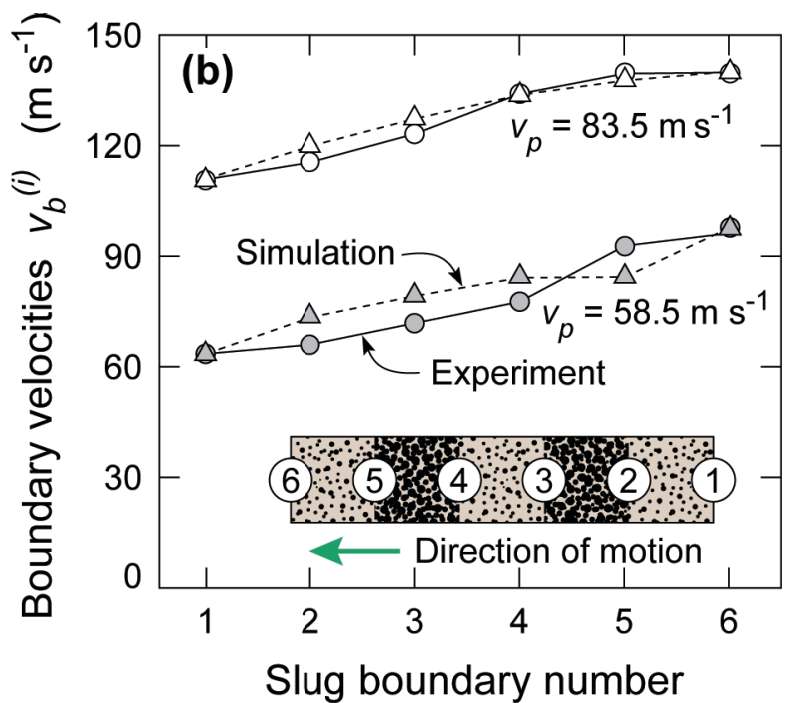

Figure 6: (a) Snapshots showing the evolution of the dry sand slug generated by a piston velocity at four selected values of time, where corresponds to the instant of the impact of projectile against the piston. (b) Measurements and predictions of the steady-state boundary velocities for two selected values of. The boundary number indicated by the superscript is illustrated in the inset of (b).

\subsection{Characterisation of transmitted momentum}

The primary goal of this study was to determine the momentum transmitted from the slug into a target whose surface properties were systematically varied. The two force transducers provide measurements of force versus time histories in the -, - and directions. The transmitted forces depend not only on the actual loads exerted by the slug but also on the location of the force measurements, structural properties of support (e.g. its stiffness and mass) and the clamping setup [33,34]. However, the integral of force history over time is less dependent on the support structure, which makes it a useful parameter to quantify the effect of impacting slugs. The force component measured by the two transducers (1 and 2) in the -direction is identified as and, respectively. Consequently, the momentum transmitted into the support structure in the -direction after a time (where corresponds to the instant that the slug first impacts the target) is given by 
and is the total momentum transmitted into the target in the -direction. Analogous expressions exist for momenta components in the other two directions (viz. and as well as and . In all experiments, to within the accuracy of the measurements (as dictated by symmetry) and hence the focus here is on the momentum measurements in the - and -direction and the resultant transmitted momentum given by

Consider the case of a dry sand slug impact at against the target covered by the Alumina plate. Temporal measurements of for a normal ) impact, Fig. 5a, (where symmetry dictates ) and both and for the inclined impact, Fig. 5b, are reported in Fig. 7. After a small initial time delay (corresponding to the time taken for the stress waves initiated at the impact location to reach the force transducers), the momentum rises sharply before oscillating about a fixed mean value at a frequency related to natural frequency of the entire setup. It is evident from Fig. 7 that for the inclined impact. In order to understand this observation, we note that if and are the resultant normal and tangential forces exerted by the sand particles on the target (Fig. 5b), momentum balance gives

and

The tangential force (from friction between the slug particles and the target) has opposite effects on and, and is the source of the separation of the two curves for the inclined impact in Fig. 7.

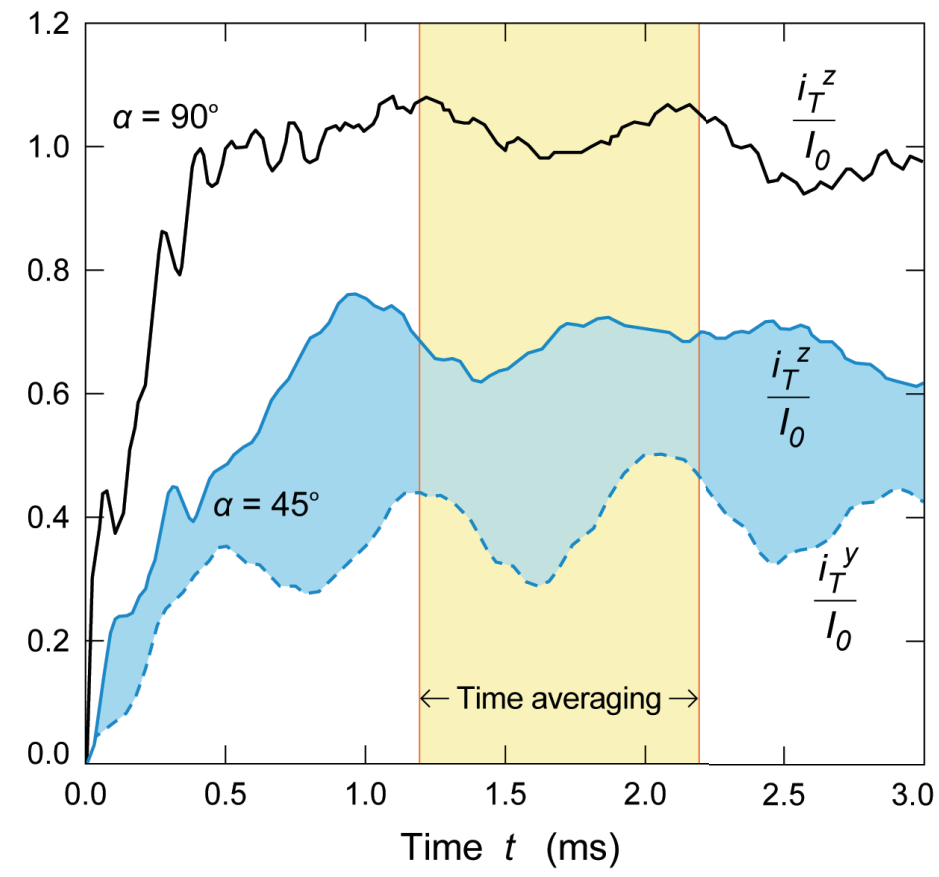


Figure 7: Measurements of the temporal variation of the normalised transmitted momentum for the normal as well as and for the inclined impacts. Time corresponds to the instant that the slug impacts the target and results are shown for a dry slug impact at against the Alumina coated target surface.

The consequences of Eqs. (2.4) and (2.5) in the limiting case when are worth further elaboration as it enables better interpretation of measurements reported subsequently. Symmetry of the normal impact () implies and. By contrast, for the inclined case we cannot independently relate and directly to without detailed information on due to the lack of symmetry in the problem. However, in the absence of friction between the particles and the target (i.e. when ), explicit relations for the transmitted momentum follow

For the case of these reduce to. These values are subsequently used as a reference during analysis of the measurements. The measurements will invariably have a finite friction between the particles and target and thus the deviations from these estimates serve to quantify the effect of friction between the particles and the target surface.

\section{Measurements for normal impact}

A montage of high-speed photographs showing the deformation of dry sand slugs as they impact normally against targets with the Alumina and sand-paper coatings are included in Figs. 8a and 8b, respectively for a slug velocity. Here, time corresponds to the instant the slug impacts the target. The slug is seen to spread laterally against the target in a mode very similar to the spreading of water jet with no visible rebound of the sand particles and no obvious differences between the two surface types. These results are consistent with observations reported by Park et al. [27] and the discrete particle computations of Pingle et al. [35]. We define the transmitted momenta and as the average values of and, respectively over a period of (Fig. 7). The measured normalised transmitted momenta are included in Fig. 9a for slug velocities in the range 73. Over the entire range of velocities, for both surface types. This measurement is consistent with the observation from the high-speed photographs that indicate (i) negligible rebound of the sand particles from the target and (ii) that the deformation modes of the sand slugs are similar in Figs. 8a and 8b. We emphasise here that the measured values of plotted in Fig. 9a are slightly greater than 1 . This is due to uncertainties in the measurements associated with the vibration of the test setup (see temporal variations plotted in Fig. 7) and not due to additional transfer of momentum into the target due to reflection of the sand.

A montage of photographs showing the normal impact of the water saturated sand slug against the Alumina coated target is included in Fig. 8c. The overall mode of deformation is similar to those of the dry sand slug in Fig. 8a and there is no measurable effect of water on the normalised transmitted momentum with for the water saturated slugs over the entire velocity range considered here (Fig. 9a). 


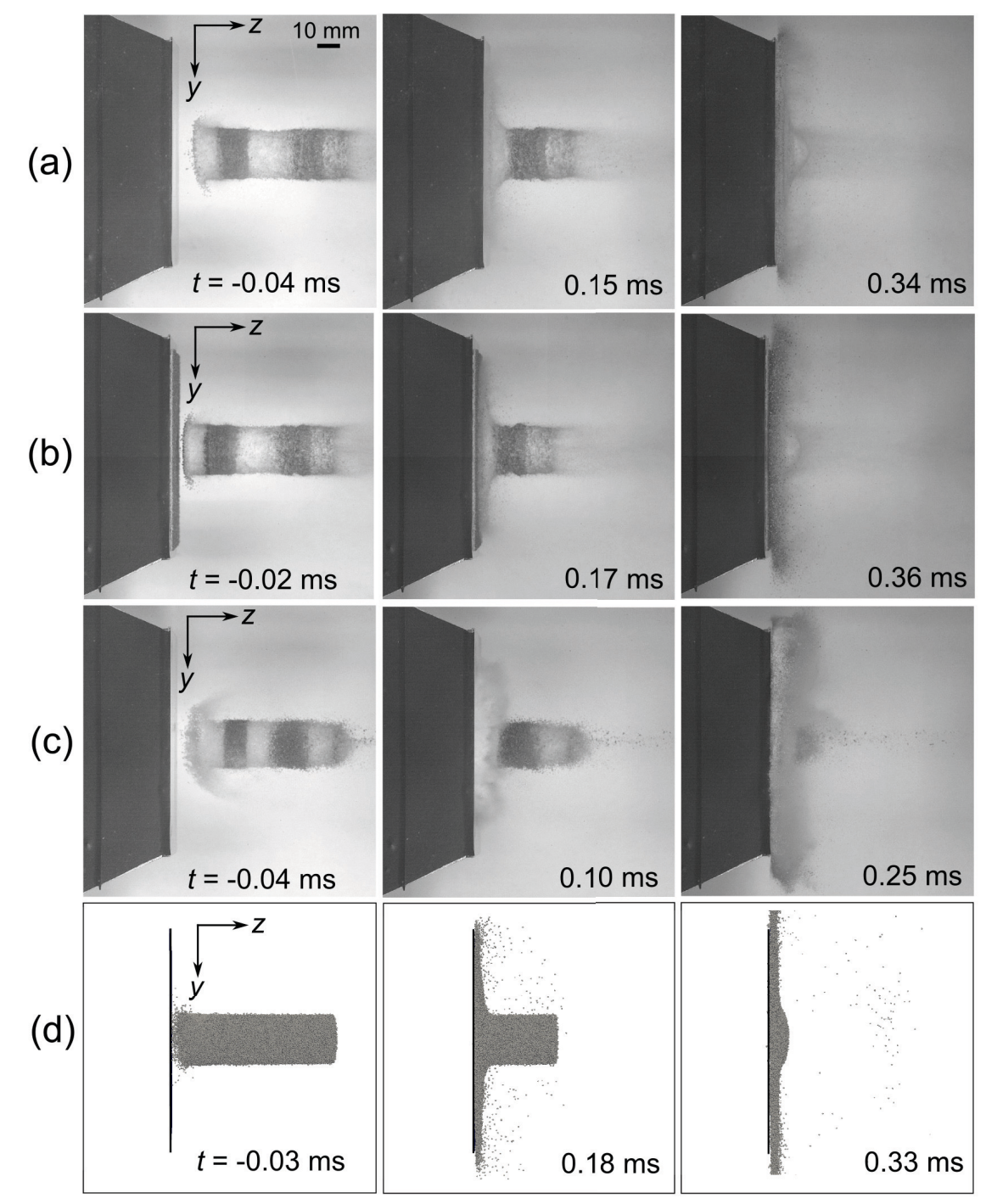

Figure 8: Montage of high-speed photographs showing the deformation of sand slugs impacting the targets in normal orientation: (a) dry sand slug against the Alumina coated target; (b) dry sand slug against the sand-paper coated target and (c) water saturated sand slug against the Alumina coated target. (d) Corresponding predictions of the dry sand slug impacting the Alumina coated target. For (a) and (b), the sand slugs have a velocity while in (c), the slug velocity. Time corresponds to the instant that the slug impacts the target. 


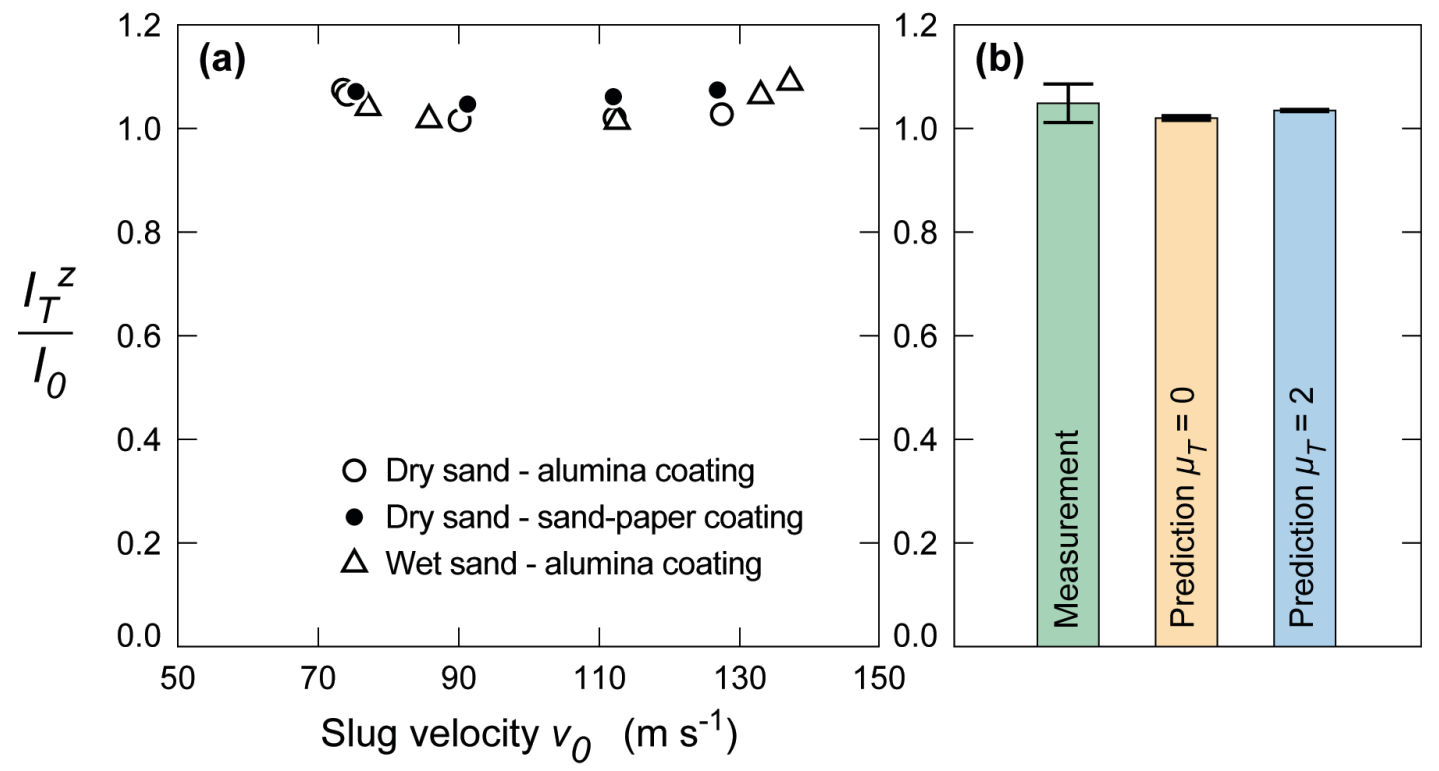

Figure 9: (a) The measured normalised transmitted momentum as a function of the slug velocity for normal impact against targets. Measurements are shown for impact of dry sand slug against the Alumina and sand-paper coated targets as well as the water saturated slug against the Alumina coated target. (b) A bar chart showing the measurements and predictions of for normal impact of the slugs. Predictions for the friction coefficient between the target and the particles and 2.0 are included and the error bars on the bars indicate the variation of the measurements and predictions over the range of slug velocities investigated here.

\section{Measurements for inclined impact}

We begin by investigating the case of the inclined target with Alumina coating and then use it as the reference case. A montage of high-speed photographs showing the impact of the dry sand slug against the Alumina coated inclined target is included in Fig. 10a. The spreading of sand particles against the target is asymmetric with both the mass of the sand particles and spreading velocity in the negative direction being less than the positive direction. This is primarily due to the fact that the momentum of the incoming slug resolves along the target surface in the positive direction. However, the flow of the sand particles along the target surface in the negative direction is caused by the reaction imposed by the target on the sand particles.

Figure 10: Montage of high-speed photographs showing the deformation of the dry sand slugs for inclined impacts against (a) Alumina, (b) PTFE, (c) Aluminium and (d) sand-paper target surfaces. In all cases, the slugs had a velocity and time corresponds to the instant the slug first impacts the target.

Normalised values of the measured transmitted momenta, and resultant transmitted momentum are included in Fig. 11a over the range of the slug velocities investigated here. Similar to the normal impact case, the fraction of the transmitted momenta are independent of over the range of velocities considered here. Moreover, the transmitted resultant momentum, . This represents approximately a 33\% reduction compared to the normal impact case where. This is primarily a result of the continued 

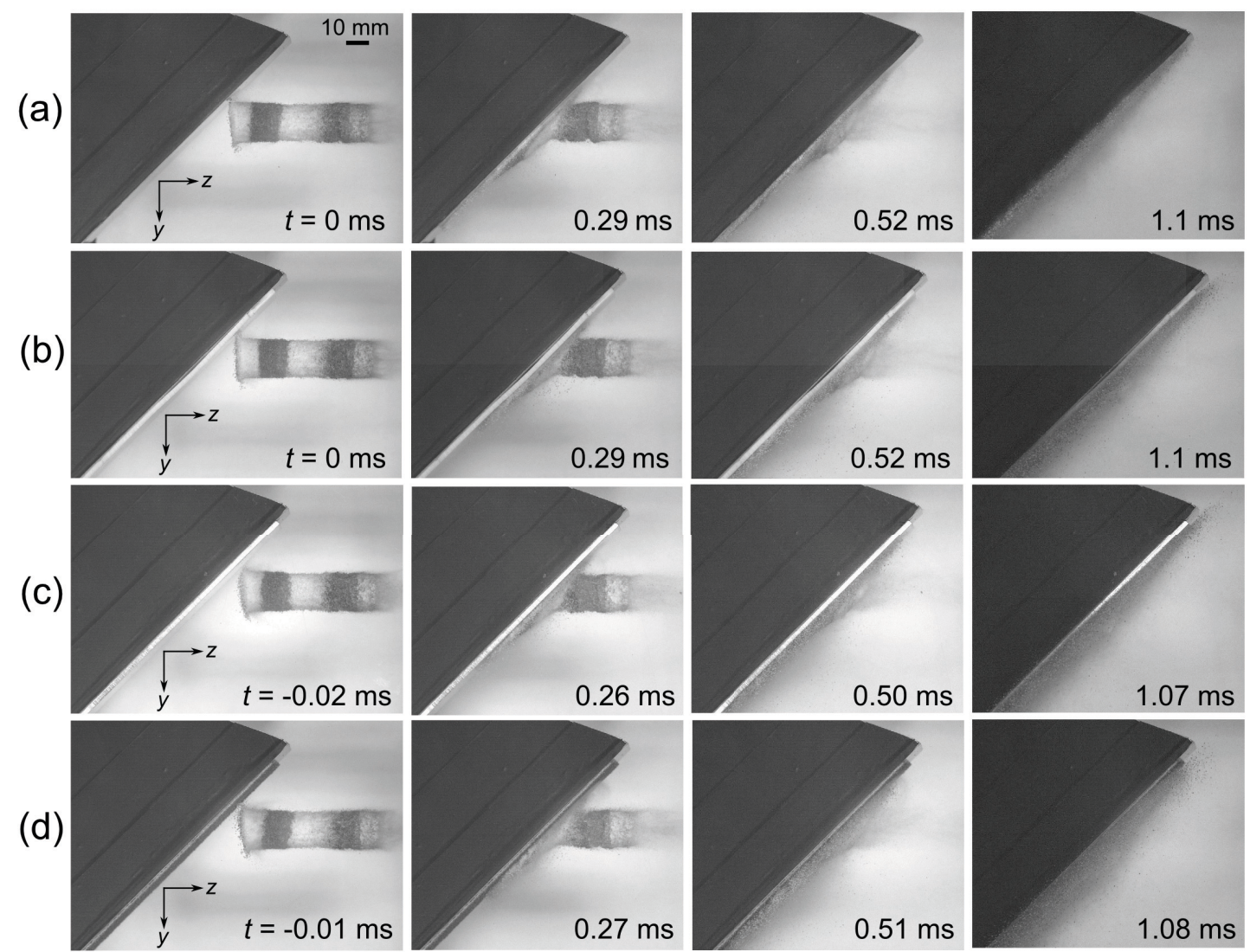

flow of the sand slug along the negative direction (i.e. retention of momentum in the slug) due to the inclination of the target surface.

However, measurements suggest that the transmitted momentum into the inclined target is higher than the predicted value in the absence of friction between the sand particles and the target surface. Recall that in the absence of friction between the sand particles and the target surface we expect that . The measurements in Fig. 11a clearly show that with and which indicates that friction between the sand particles and the target surface substantially affected the transmitted momentum even in this reference case of the Alumina coated target. Furthermore, the resultant transmitted momentum , which represents a $9 \%$ increase over the value predicted in the absence of friction between the target surface and the sand particles.

Measurements of transmitted momentum for the impact of the water saturated sand slugs against the inclined target with the Alumina coating are also included in Fig. 11a. Again, there is no effect of the slug velocity on the normalised components of all the transmitted momenta and moreover, the measurements of the normalised momenta of the dry and water saturated slugs are identical to within experimental accuracy. We thus conclude that water saturation does not affect the mechanism of momentum transmission to the target and all subsequent results are presented for only the dry sand slugs. 


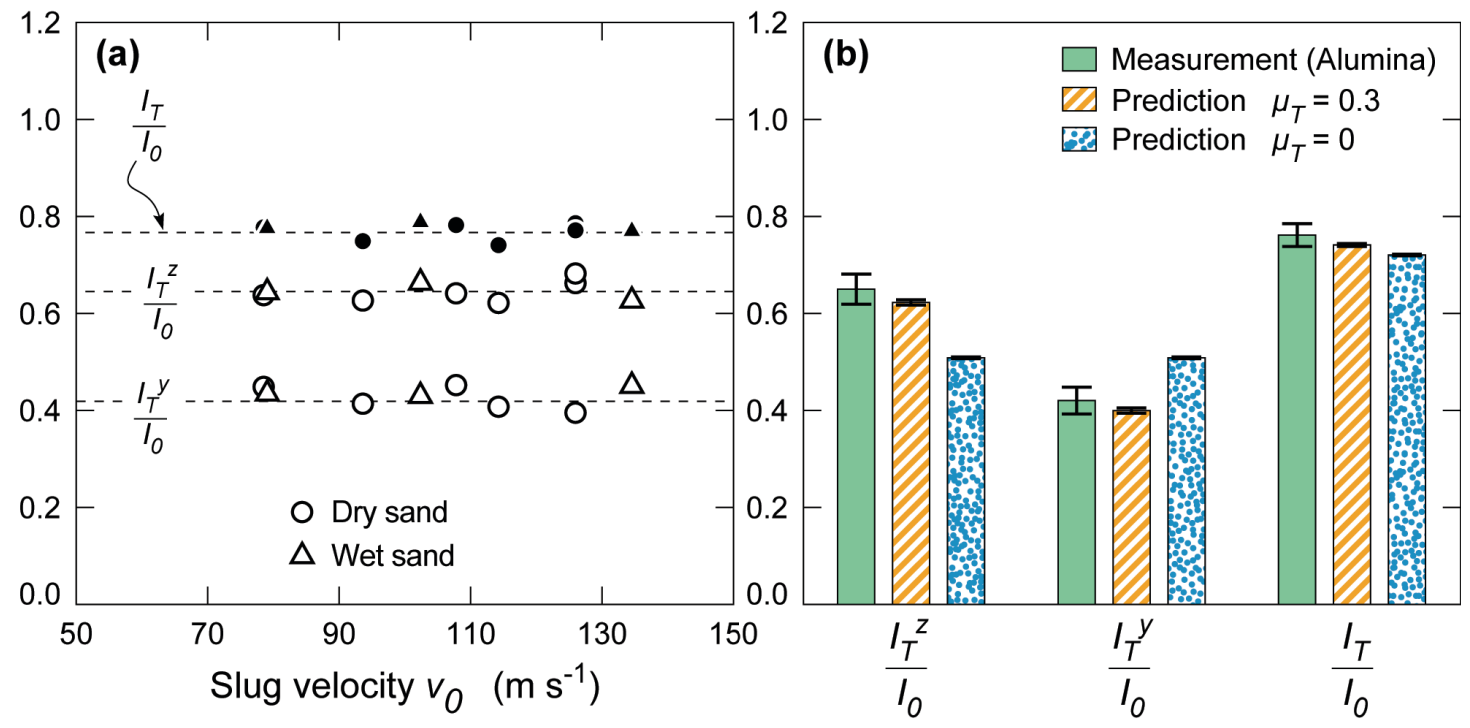

Figure 11: (a) The measured normalised transmitted momenta, and as a function of the slug velocity for inclined impact of dry and water saturated slug against the Alumina coated target. (b) A bar chart showing the measurements and predictions of, and for inclined impact of the dry sand slugs against the Alumina coated target. Predictions are shown for the friction coefficient between the target and the sand particles and 0.3 . The error bars indicate the variation of the measurements and predictions over the range of slug velocities investigated here.

\subsection{Effect of surface type}

Frictional forces between the sand particles and the target surface play an important role in modulating the transmitted momentum for the inclined impact case as shown above. In order to better understand the relation between surface type and the transmitted momentum, the transmitted momentum was investigated for three other surface coatings: (i) PTFE which is known to reduce adhesion but has a low hardness and relatively high surface roughness; (ii) Aluminium which has a low surface roughness but also a relatively low hardness and (iii) sand-paper with a high surface roughness and low hardness. The transmitted momenta for these surfaces is then compared with that of the Alumina surface with relatively low surface roughness and high hardness.

A montage of high-speed photographs showing the deformation of the dry sand slug impacting against the inclined target with PTFE, Aluminium and sand-paper coatings are shown in Figs.10b, 10c and 10d, respectively. There were no observable differences in the deformation modes of the slugs. By contrast, the transmitted momenta were strongly dependent on the surface type as summarised in the bar chart in Fig. 12. This figure shows the measured average values of, and with the error bars indicating the range of values obtained over the slug velocities investigated here. We emphasise that no trend with was observed here similar to the results shown in Figs. 9a and 11a. Instead, the error bars indicate the variability in the experimental measurements. The resultant transmitted momentum for the target surfaces increases in the order Alumina to PTFE to Aluminium to sand-paper though even for the sandpaper case is less than the corresponding value for a normal impact. Correspondingly, the transmitted momentum increases in the same order as while decreases. This is emphasised in Fig. 12, where we also include a bar chart summarising the 
measurements of for the four surface coatings. These measurements clearly show the effect of the surface type on the levels of transmitted momentum. For example, increased by $25 \%$ and by $11 \%$, in changing from Alumina to sand-paper coating.

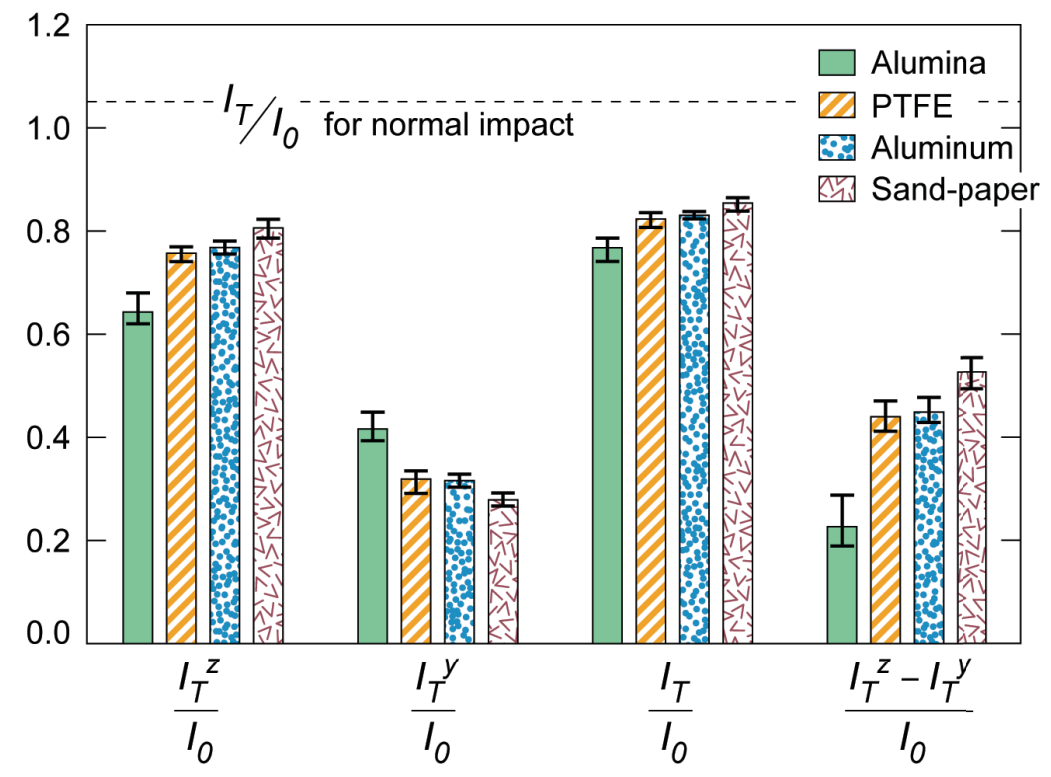

Figure 12: Bar charts summarising the measured values of,, and for inclined impact of the dry sand slugs against targets with the four different surface coatings. The error bars indicate the variation of the measurements over the range of slug velocities investigated here. For reference we have indicated the normalised resultant momentum for normal impact.

The differences are attributed to frictional interactions between the sand particles and the surface of the target. To provide further insight into these differences Figs. 13a, $13 \mathrm{~b}$ and $13 \mathrm{c}$ show post-test photographs of the PTFE, Aluminium and sand-paper surfaces, respectively after impact by dry sand slugs. The local co-ordinate system along the surface of the target is defined in Figs. 13a and 13d, and is oriented such that the primary spreading of the slug occurs along the positive direction. Significant embedding of the sand particles into the surface coatings is observed and this embedding increased in the order PTFE to Aluminium to sand-paper. No photograph of the Alumina surface is included as no embedding or any post-test visual indication of the impact of the sand slug was observable for that surface. The measured increases with the level of observed embedding of the sand particles. The embedding of the particles is assumed to increase the effective friction between the sand particles and the target surface. Since embedded particles are also fully brought to rest, all their momentum must be transferred to the target.

The measurements reported here demonstrate the importance of the surface properties in governing the transmitted momentum for sand slug impacts with inclined targets. While the level of friction between the particle and the target surface clearly modulates the transmitted momentum, the relationships between surface material properties and the frictional interaction with the granular slug is as yet unclear. The results presented here suggest that the initial surface roughness is not a critical parameter (e.g. the Aluminium surface has a lower compared to Alumina but results 
in a higher ). Rather, surface properties that govern the embedding of particles into the surface play the crucial role. These properties are hardness and adhesion. For example, the reason the Alumina surface seems to minimize friction and thereby is that its high hardness implies there is no embedding of sand particles at the impact velocities considered.

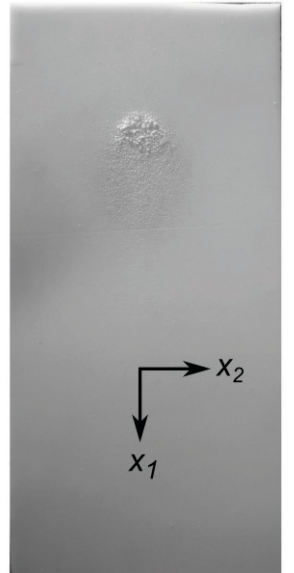

(a) PTFE

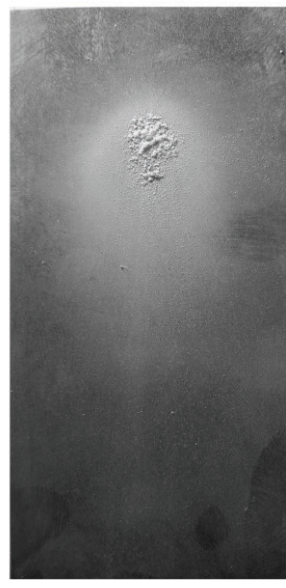

(b) Aluminium

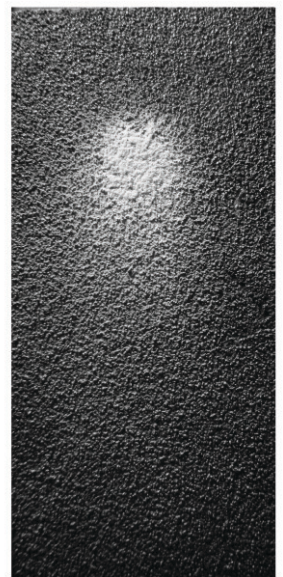

(c) Sand-paper

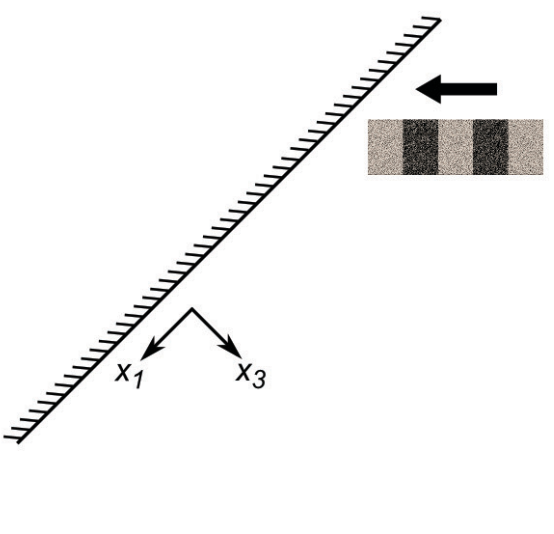

(d)

Figure 13: Post-test photographs showing the impacted surfaces of the (a) PTFE, (b) Aluminium and (c) sand-paper coatings subjected to inclined impacts by dry sand slugs. (d) A sketch showing a side view of the impact of the sand slug against the inclined target. The sketch indicates the co-ordinate system used in (a)-(c) to clarify the direction of the impact and the consequent flow of sand particles.

\section{Discrete particle simulations and comparisons with measurements}

The experimental measurements reported above show the effect of surface type on the level of transmitted momentum for both normal and inclined sand slug impacts. Numerical calculations are now utilized to better understand the interactions of sand slugs with the targets. This will enable quantification of the role of friction between the sand particles and the target that was shown experimentally to modulate the transmitted momentum in the inclined impact case.

\subsection{Simulation methodology}

The impact of dry sand slug against stationary targets was modelled using a coupled discrete particle/Lagrangian finite element simulation scheme. In this approach, the sand particles were modelled as discrete spherical particles using the GRANULAR package in the multi-purpose molecular dynamics code LAMMPS $^{5}$ [36] while the rigid targets were modelled within the Lagrangian commercial finite element package Abaqus ${ }^{6}$. These two modelling schemes were coupled using the $\mathrm{MpCCI}^{7}$ interface as described below. The modelling scheme therefore consisted of four steps: (i) the

\footnotetext{
5 http:// lammps.sandia.gov/

${ }^{6} \mathrm{http}: / / \mathrm{www} .3 \mathrm{ds} . \mathrm{com} /$

${ }^{7}$ http://www.mpcci.de/
} 
discrete particle approach to model the sand particles; (ii) generation of the highvelocity slug due to the pushing of the slug out of the launcher by the piston; (iii) Lagrangian finite element scheme to model the contact between the target and the particles; (iv) an MpCCI interface for coupling between the discrete particle and FE schemes. Effects of gravity and air drag are neglected in the simulations. (At the relatively low velocities considered here, we are in a Stokes drag regime where the reduction in the velocity of the particles over the timescales of the experiments is negligible.)

Three-dimensional simulations are performed using mono-sized spherical particles of diameter and mass . The soft-particle contact model (Fig. 14), introduced by Cundall and Strack [37], and extended to large scale simulations by Campbell and Brennen [38] and by Campbell [39], accounted for both inter-particle interactions as well as the interactions of the particles with the target surface. The interparticle contact law comprises:

(i) A linear spring with spring constant and linear dashpot with damping constant connected in parallel, governing the contact force-displacement relation in the direction connecting the particle centres.

(ii) A linear spring of constant and Coulomb friction coefficient connected in series, governing the tangential contact relationship.

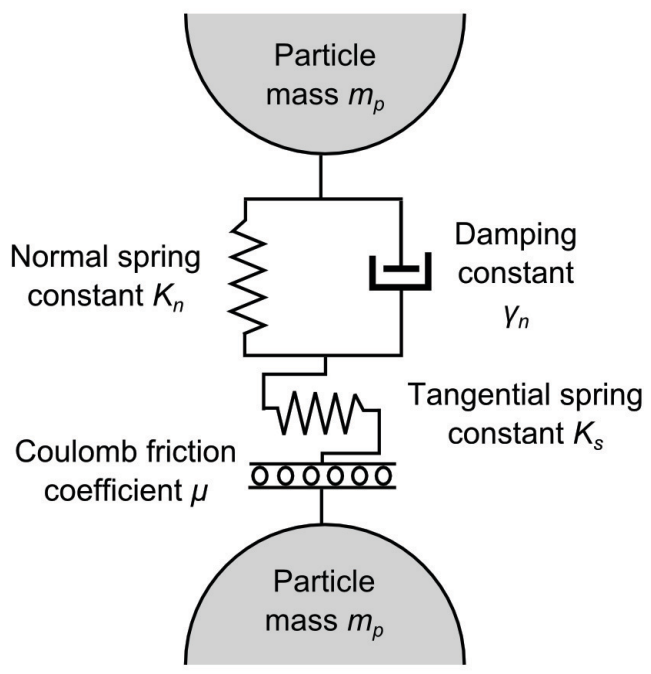

Figure 14: Sketch of the soft inter-particle contact model between spherical particles of diameter used to simulate the sand particles.

With as the distance of separation of the particle centres, and the particle interpenetration given by, the normal force during active contact () is given by

where is the reduced mass of the two contacting bodies. For impacts between a pair of particles, while for impacts between a particle and the target, . 
The tangential force only exists during an active contact, and opposes sliding. It is limited in magnitude to, where is the friction coefficient as follows. If is defined as the tangential displacement rate between the contacting particles, can be represented by an elastic-plastic relation of Coulomb type with stiffness ,

The critical difference for interactions between particles and between a particle and the target is the coefficient of friction which will be different for the two cases. Here, and refer to the value of for inter-particle and particle/target interactions respectively. The value of damping constant dictates the loss of particle kinetic energy during normal collision and is directly related to the coefficient of restitution according to

The collision time for individual binary collisions then follows from (5.1) as

and thus in the limit of plastic collisions with, the contact time . Newton's equations for both the translational and rotational motions of the particles were integrated using a Verlet time-integration scheme (Newark-Beta with ). The time-step for integration was taken to be less than in order to ensure accurate integration of the equation of motions given by and, where is the angular acceleration of the particles with their $2^{\text {nd }}$ moment of inertia about the diametrical axis.

The rigid targets (dimensions identical to those used in the experiments) were modelled as analytical rigid surfaces in Abaqus finite element analysis code, and all the six degrees of freedom ( 3 rotational and 3 translational) were constrained to prevent all rigid body motions. The coupling between the LAMMPS discrete particle and the Abaqus finite element calculations was carried out via an MpCCI Code adapter API as follows. At any time, suppose that a proportion of the particles are in contact with the target surface. Consider one such particle in contact with the target surface. The interpenetration is defined as, where is the distance between particle centre and contact point on the target (note the difference in the definition of for contacts between particles and between the particle and the target). The rate is the relative approach velocity of the particle and the point of contact on the target surface, and likewise is the tangential velocity. The normal and tangential contact forces are calculated using Eqs. (5.1) and (5.2). These forces were then added as nodal forces to the appropriate surface elements in the Abaqus finite element calculations to complete the coupling between the discrete and finite element calculations. The transmitted momentum was calculated by time integrating the appropriate reaction force on the target; e.g. to calculate we temporally integrate the reaction force in the direction on the target over a sufficiently large time period so as to obtain the steady-state value of . 
The following reference properties were assumed in all the calculations. The granular slug was modelled with spherical particles of diameter made from a solid of density (equal to that of Silica). The particle contact model was defined by four parameters, , and. While Liu et al. [2] demonstrated that these parameters do not affect the interaction response of the granular slug impacting beams, Goel et al. [40] demonstrated that strongly influence the evolution of the granular slug as it emerges from the launcher. It was therefore necessary to calibrate, as discussed below, while maintaining the other parameters fixed at reference values based on previous studies; see for example Refs. [2,35,41]. The reference values of the parameters are , $[42,43]$, and. The friction coefficient between the sand particles and the target surface depends on the surface type and is unknown. Thus, parametric studies for the sensitivity of the results to this parameter are presented. Frictional interactions between the cylindrical cavity surface of the launcher in contact with the sand particles were neglected.

The sand slug ejected from the launcher had an axial particle velocity gradient as seen in Fig. $6 \mathrm{~b}$ that resulted in the lengthening of the slug during free-flight. Goel et al. [40] demonstrated that this velocity gradient is strongly dependent on and hence needed to be calibrated independently. This was performed as follows. Following Uth et al. [29], sand particles in a cylindrical cavity were subjected to constrained compression as sketched in Fig. 15a. The measured applied axial stress (defined as ratio of the applied compressive force to the cross-sectional area of the cylindrical cavity) versus nominal strain (defined as the ratio of the relative displacements of the two pistons to the initial height of the granular assembly within the cylinder) is plotted in Fig. 15b for an applied strain rate. This data can be used to calibrate the inter-particle stiffness as follows.

First, the spherical sand particles of the numerical model were packed into a rigid cylindrical cavity as sketched in the inset in Fig. $15 \mathrm{~b}$ to create a granular assembly of dimensions identical to that used in the experiment shown in Fig. 15a. The spherical particles had an initial volume fraction (prior to the application of the compressive force) of 0.61. Simulations of the compressive response were conducted by compressing the granular assembly via a rigid piston as shown in Fig. 15b at a displacement rate (corresponding to a nominal strain rate ). The predicted nominal stress versus nominal strain responses are plotted in Fig. 15b for three choices of with the remaining contact parameters kept fixed at their reference values. A contact stiffness brought the predictions in close agreement with the measurements and was therefore used as the reference value for ${ }^{8}$.

\footnotetext{
8 The friction coefficient, shear stiffness and damping do not affect the predicted constrained compressive response shown in Fig. 15b over an applied strain rate range .
} 
(a)

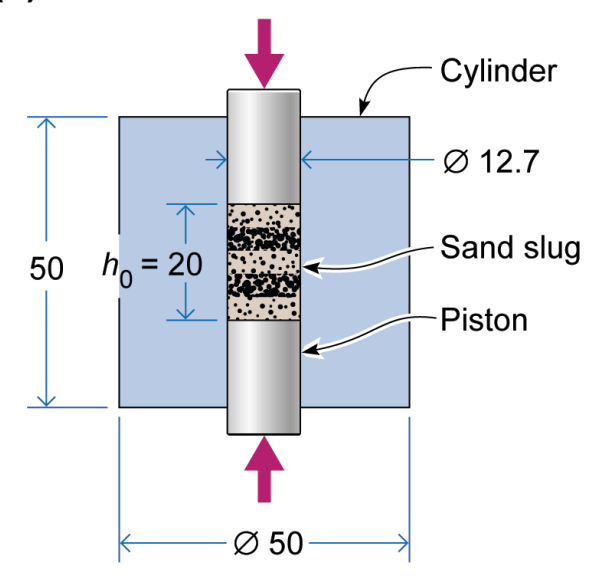

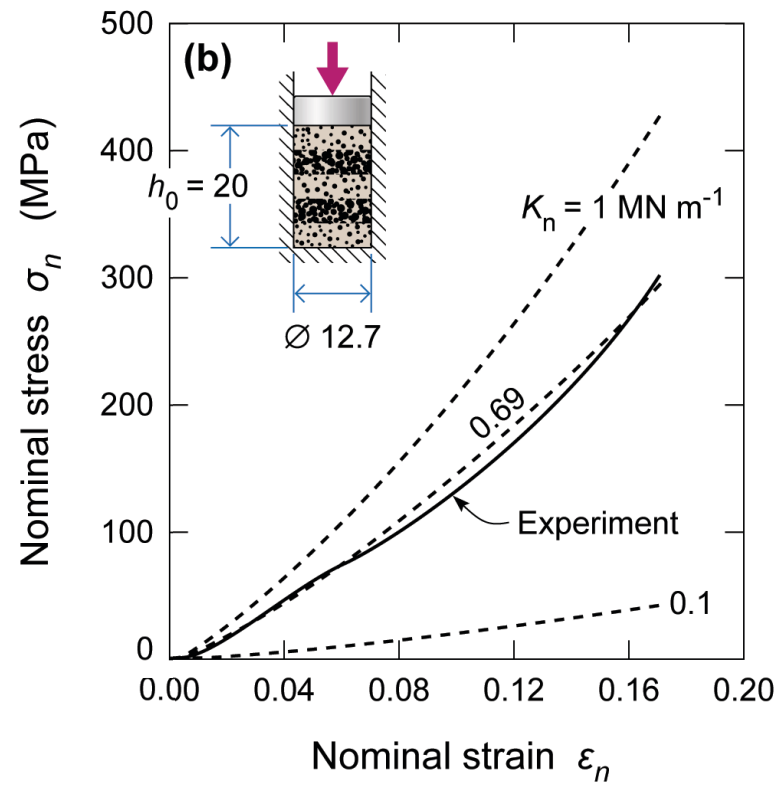

Figure 15: (a) Sketch of the experimental setup used to measure the constrained compression response of the sand slug. (b) Comparison between measurements and predictions of the constrained compression response. Predictions are shown for three selected values of the normal inter-particle contact stiffness . The setup used in the simulations is shown as an inset. All dimensions are in $\mathrm{mm}$.

\subsection{Generation of the sand slug}

To accurately capture the state of the sand slug during its free-flight and thereby its state just prior to impact, it is necessary to explicitly model the ejection of the sand slug from the launcher as described in Goel et al. [40]. In order to simplify the numerical calculations, the impact of the projectile against the piston and the deformation of the piston were not directly modelled. Rather, the piston was modelled as a rigid body as shown in Fig. 16a and given a velocity, motivated from the data in Fig. $4 \mathrm{~b}$ in a manner similar to that of Goel et al. [40]. Thus, following Goel et al. [40], it suffices to approximate as

where is the time taken for the piston to ramp up to its final velocity .

Simulations of the ejection of the sand slug from within the launcher were performed as follows. First the spherical sand particles were inserted into a cylindrical cavity of inner diameter capped at one end by a rigid piston as shown in Fig. 16a. The compacted slug had a length and the free end of the slug needed to travel a distance of $10 \mathrm{~mm}$ to emerge from the launcher, consistent with the experimental design. The piston was then imparted a velocity versus time history specified by Eq. (5.5) which pushed the slug out of the launcher. The ramp time was calibrated to ensure agreement between the measured and predicted boundary velocities; see for example Fig. 6b. This involved obtaining a value of for each piston velocity employed in this study: this calibrated relation is plotted in Fig. 16b. 
The impact of the slugs generated in this manner against the targets is then solved via the coupled discrete particle/Lagrangian method with the target placed at a stand-off in line with the experimental setup.

(a)

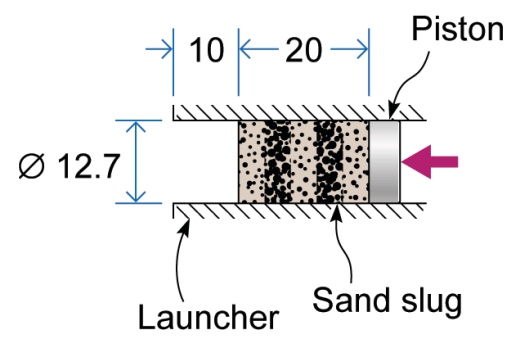

$$
\begin{aligned}
& \text { All dimensions in } \mathrm{mm} \\
& \text { - Not to scale - }
\end{aligned}
$$

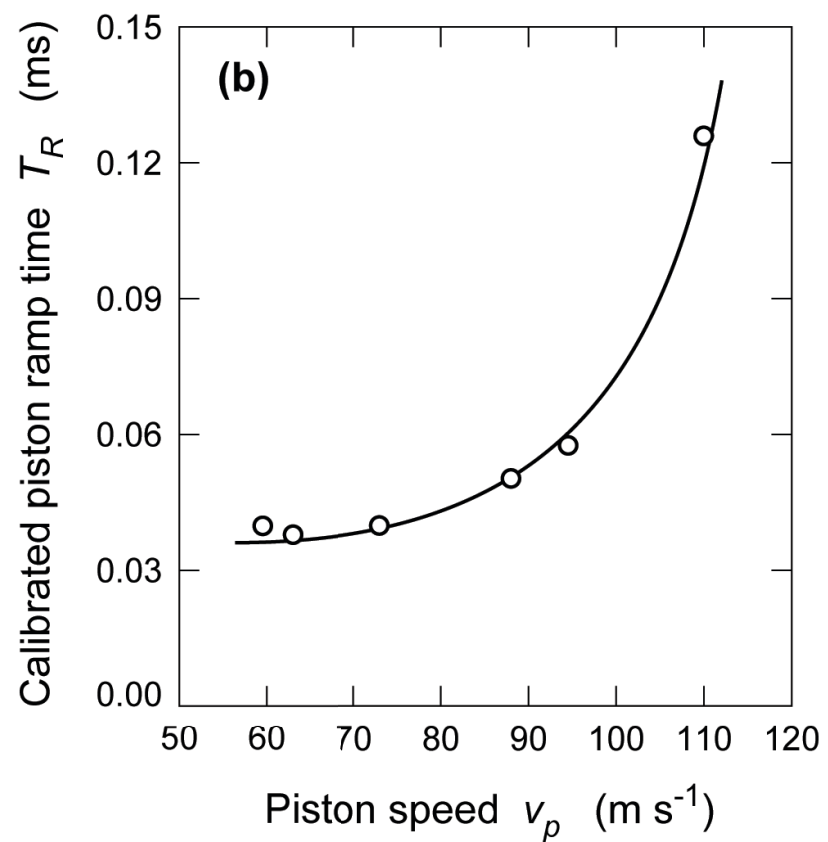

Figure 16: (a) A side view sketch of the launcher/piston setup used in the numerical simulations. (b) The calibrated piston ramp time versus piston velocity relation that accurately reproduces the measured spatial distribution of particle velocities in the sand slug during free-flight.

\subsection{Comparison with measurements}

Snapshots showing predictions of the spreading of a normally impacted sand slug are included in Fig. 8d for three selected times, . The friction coefficient between the particles and the target was taken to be in this simulation. Overall, the predicted spreading process appeared consistent with the dry sand impact high-speed photographs for the Alumina and sand-paper coated target surfaces in Figs. 8a and 8b, respectively. Next, consider the predictions of the transmitted impulse. Similar to the measurements, and was approximately independent of . Thus, it suffices to compare the measured and predicted average values of only over the range of investigated here. This comparison is shown in the bar chart in Fig. $9 \mathrm{~b}$ for predictions with and a large friction coefficient of . In Fig. 9b, error bars are used to indicate the variation of the predictions with - this variation is negligible for the simulations, and hence the error bars are barely discernible. It is evident that: (i) the predictions are in excellent agreement with the measurements, and in all cases indicating negligible rebound of the sand particles and (ii) the friction coefficient between the particles and target has no discernible effect on the transmitted momentum; consistent with the experimental observation that values of the Alumina and sand-paper target surfaces were the same (Fig. 9a). While does not affect any of the transmitted momentum components or , the tangential forces between individual particles and the surface in the and directions do increase sharply with increasing. However, the symmetry of the impact leads to these forces being cancelled out with the result that . The lack of such symmetry in 
the inclined impact case examined next is therefore expected to introduce a strong dependence of the transmitted impulse on .

Snapshots from the simulation of the slug impacting the inclined target with are shown in Fig. 17. Two views are shown in order to better illustrate the spreading of the slug over the target: in Fig. 17a, a side view showing the plane (as observed with the high-speed photographs) is included while in Fig. 17b, we show an orthographic view perpendicular to the target surface (i.e. the plane as defined in Figs. 13a and 13d). The sand particles are seen to spread primarily in the positive direction consistent with the high-speed photographs in Fig. 10 (though a view showing the spreading in the direction is not available from the experiments). Again, like the measurements, the normalised transmitted momenta are independent of and thus comparisons between measurements and predictions of, and for impact against the Alumina coated target are shown via a bar chart in Fig. 11b similar to Fig. 9b. In Fig. $11 \mathrm{~b}$ predictions for and are included. The frictionless case overpredicts and underpredicts as well as but the measurements and predictions are in good agreement using. Thus, unlike in the normal impact case the momentum predictions are sensitive to with the simulations suggesting significant frictional forces between the sand particles and the surface even for the Alumina coated case: the surface anticipated to have the lowest frictional effects.

(a)

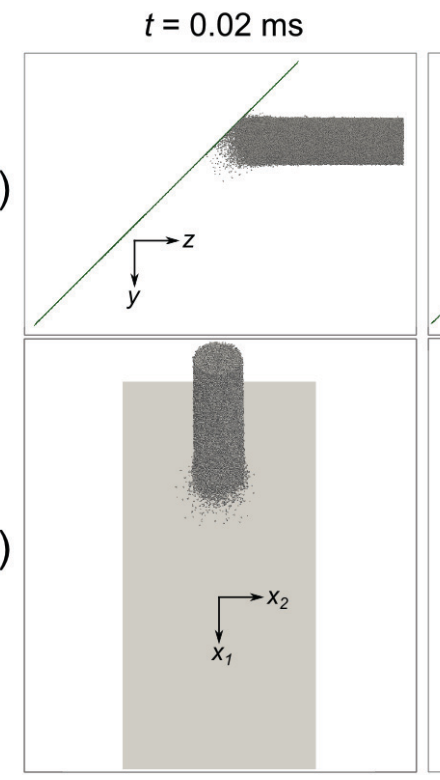
$0.27 \mathrm{~ms}$ $0.52 \mathrm{~ms}$ $1.07 \mathrm{~ms}$

(b)
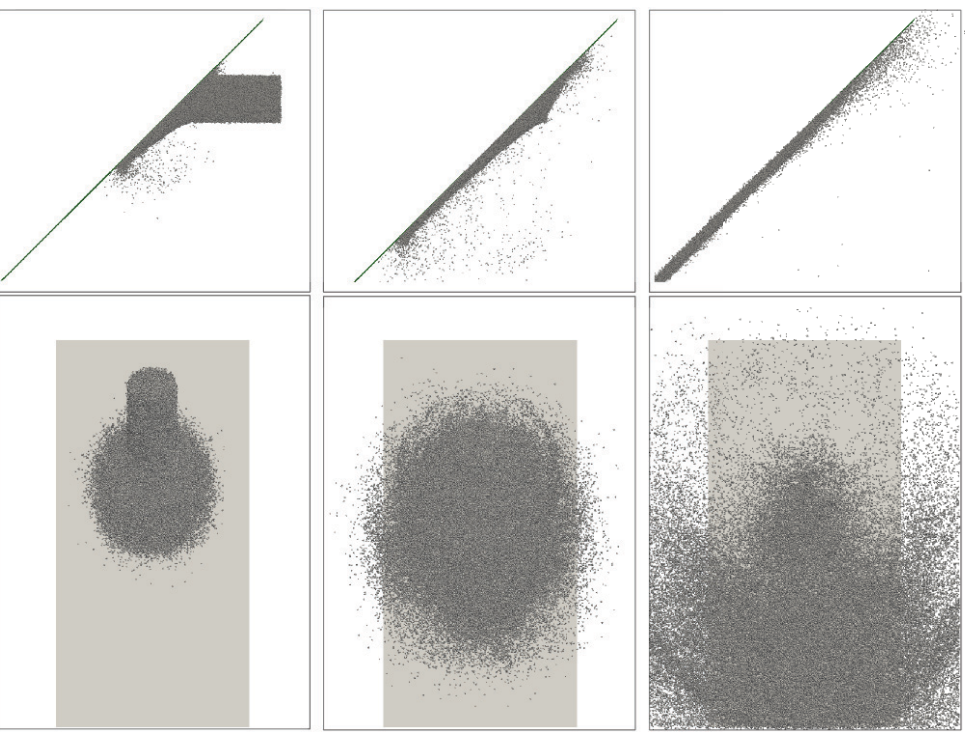

Figure 17: Snapshots from the simulations of the impact of the slug against the inclined target with . The snaphots are shown at the same time instants as the high-speed photographs in Fig. 10 with time corresponding to the instant the slug impacts the target. (a) A side view showing the plane as seen in the high-speed photographs in Fig. 10 and (b) an orthographic view perpendicular to the target surface (i.e. the plane as defined in Figs. 13a and 13d).

Frictional forces between the sand particles and the target increased and decreased with increasing as the impact surface was changed from Alumina to PTFE to Aluminium and finally to sand-paper as seen in Fig. 12. It is thus instructive to examine the sensitivity of the predictions of to and these results are presented in Fig. 
18 where in addition to the reference value of inter-particle friction coefficient (, we show predictions using and 2.0. Recall that in the absence of friction between the particles and the target (i.e.), momentum conservation dictates that and thus when but increases with before saturating beyond. The simulations in Fig. 18 indicate that the inter-particle friction has no effect on at low but shows a mild sensitivity to at high values of . For example, increases from approximately 0.3 to about 0.35 as is increased from 0.6 to the unrealistically high value of 2.0. The measured values of for the Alumina and sand-paper coated target surfaces are indicated in Fig. 18. We note that while a combination of and could be found to force the simulations to fit the measurements for the Alumina surface, no combination of friction coefficient values were able to bring the predictions into agreement with the measurements for impact with the sand-paper surface. Such a discrepancy also holds for the PTFE and Aluminium surface coatings.

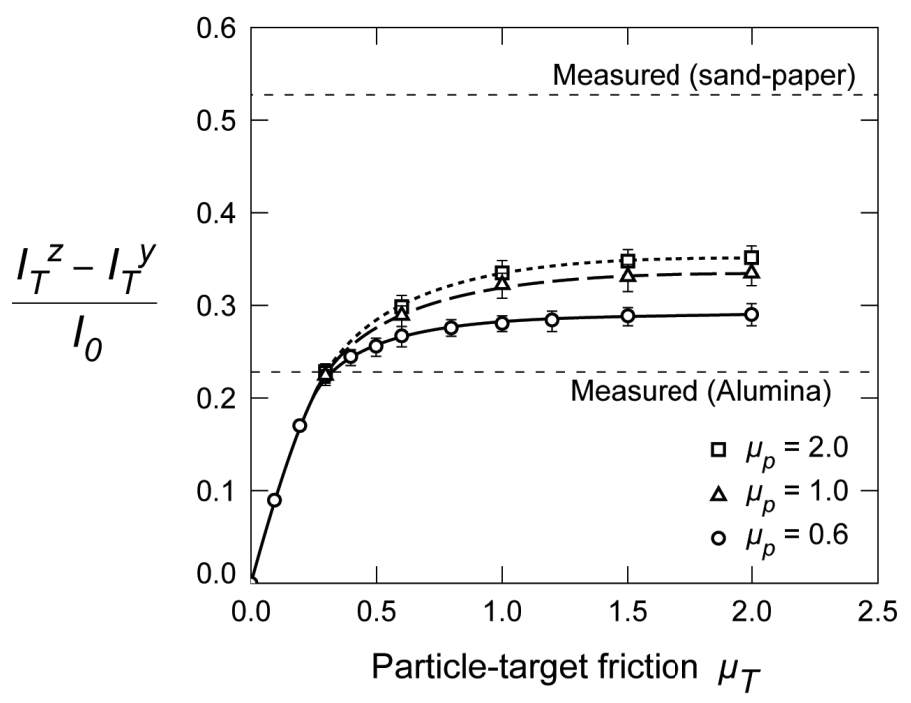

Figure 18: Predictions of the dependence of the transmitted momentum on the friction coefficient between the sand particles and the inclined target surface. Results are shown for three values of the inter-particle friction coefficient. The averaged measured values of for impact of the dry sand slug against the inclined target with the Alumina and sand-paper coatings are also indicated.

The transmitted momentum predicted for the inclined impact is insensitive to any of the other inter-particle contact parameters, consistent with observations for the normal impact case by Pingle et al. [35] and by Liu et al. [2]. Thus, there seems to be a fundamental discrepancy between predictions and measurements. This discrepancy increases when frictional forces between the particles and the target surface increase, i.e. discrepancies are highest for the sand paper coating which imposes maximum constraint to the flow of the particles.

\section{Sources of the discrepancy between simulations and measurements}

The critical conclusion from Fig. 18 is that the simulations are unable to capture the interactions between the sand particles and the target accurately when frictional interactions between the particles and the target surface are high. In order to understand this issue, we consider a simpler setting in which the sand slugs have a 
spatially uniform density characterized by a particle volume fraction . All particles in such slugs now have the same velocity in the direction with the other two translational velocities, and all rotational velocities set equal to zero just prior to impact. Recall that the slug velocity does not affect the normalised transmitted momentum and thus all simulations presented in this section were performed using . Parametric studies of the effect of the inter-particle contact parameters are presented but unless otherwise specified, these values are set equal to the reference values specified in Section 5.1 with. In addition, the reference particle volume fraction of particles in the slug was approximately equal to that for the slugs in the experiments just prior to impact.

\subsection{Role of particle and slug properties}

The role of particle contact stiffness, coefficient of restitution and particle diameter in modulating the momentum transfer for the impact of granular slugs against the inclined targets is first investigated. In each case, while the parameter being investigated was varied, all other parameters were held fixed at their reference values. Calculations were performed for, and. These ranges bound the uncertainty in these parameter values for the sand slugs. The components of the transferred momentum were not affected (changes were less than 1\%) for simulations using these ranges of the parameters, and was consistent with previous studies. For example, Pingle et al. [35] and Guttenberg [44] demonstrated that does not affect the flow of granular jets as increasing results in more collisions per unit time so that the total dissipation remains approximately constant. Similarly, while Cheng et al. [30] have shown that as the number of particles across the width of the a granular jet is reduced, the jet stops behaving as an aggregate of particles and more like individual non-interacting particles. However, even with the largest particle diameter of (for which the slug contained the smallest number of particles) this limit was not approached.

The sand slug in the experiments lengthened in free-flight, and thus the particle volume fraction in the slugs was constantly evolving. While the simulations in Section 5 appear to adequately capture the velocity gradients within the slug, there is always some room for uncertainty. In order to investigate whether such uncertainty in could affect the predictions of the transmitted momentum, the role of was investigated by performing impact simulations using sand slugs with . All these slugs were of diameter $12.7 \mathrm{~mm}$ with reference particle properties and was tuned by changing the length of the slug (the particles were still randomly distributed over the slug volume). Predictions of the variation of and with are included in Fig. 19 for the reference friction coefficient values and. An additional set of predictions with very high friction coefficients are also included. The increase in the friction coefficients slightly increases both and. Further, decreasing also marginally increased the transmitted momenta as the impact changed from the case of an impact of an aggregate of sand particles to individual particle impacts as discussed in Ref. [35]. However, these effects were small and are insufficient to explain the large discrepancies between predictions and measurements for the inclined impact of the sand-paper coated target surfaces reported in Section 5. 


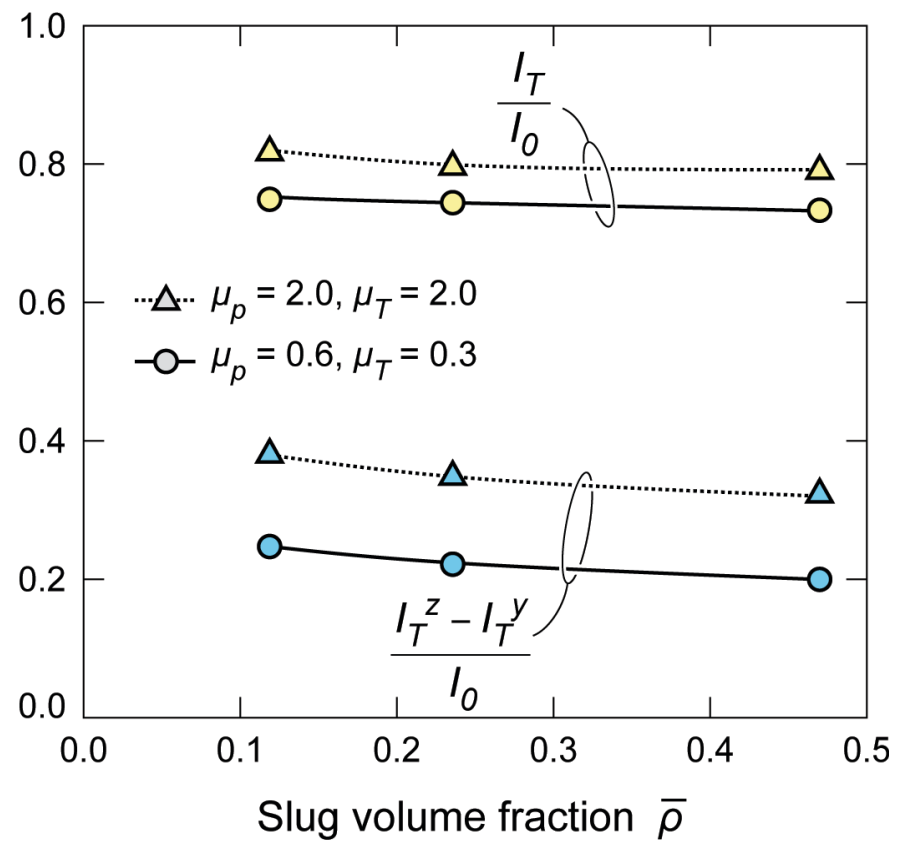

Figure 19: Predictions of the variation of the transmitted momenta and with the volume fraction of sand particles within the uniform density slug just prior to impact against the inclined target. Results are shown for the reference inter-particle and particle/target friction coefficients and, respectively and an additional set of calculations with high values of and .

\subsection{Effect of rotational inertia}

The sand particles comprising the slugs are sub-spherical in shape (Fig. 3) while in the simulations they are modelled as perfect spheres of diameter. The tendency of particles to rotate is expected to decrease with increasing angularity; see for example Refs. [45,46]. To investigate the role of particle rotation on the transmitted momentum for inclined impact of the slug, it is noted that the rotation of particles is governed by their $2^{\text {nd }}$ moment of inertia, $J_{s}$ about their diametrical axis which is given by

where is the radius of gyration of the sphere. In order to explore the role of particle rotation, we maintain the spherical shape of the particles but artificially change the radius of gyration from to and study the sensitivity of the transmitted momentum to the ratio. Such calculations are artificial in the sense that while contact between particles and particles and the target is detected based on spherical particles of diameter, the rotational motion of the particles is obtained by integrating Newton equations for the individual particles with a $2^{\text {nd }}$ moment of inertia about the diametrical axis.

Predictions of and as a function of are included in Figs. 20a and 20b, respectively for 5 choices of in the range 0.1 to 1.0 with. Both and increase significantly with increasing for, i.e. decreasing the tendency of the particles to rotate strongly affects the transmitted momentum when there is a high friction coefficient between the target surface and the particles. In fact for in the range 15 to 50 and, the predicted values of and seem to lie in the range measured for the sand-paper coated targets. 


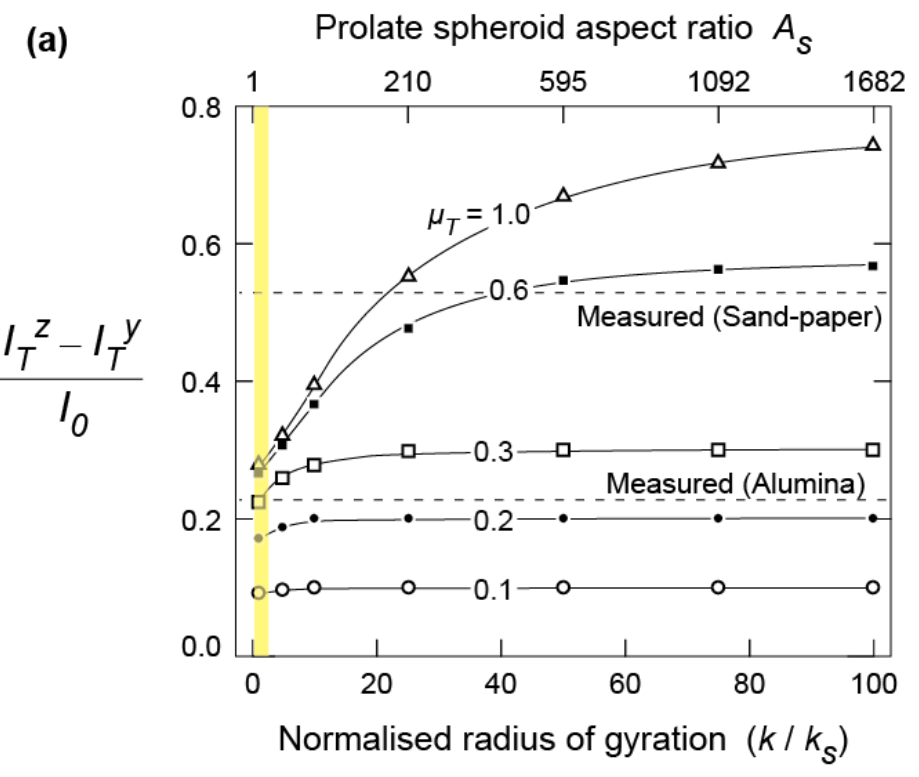

(b)

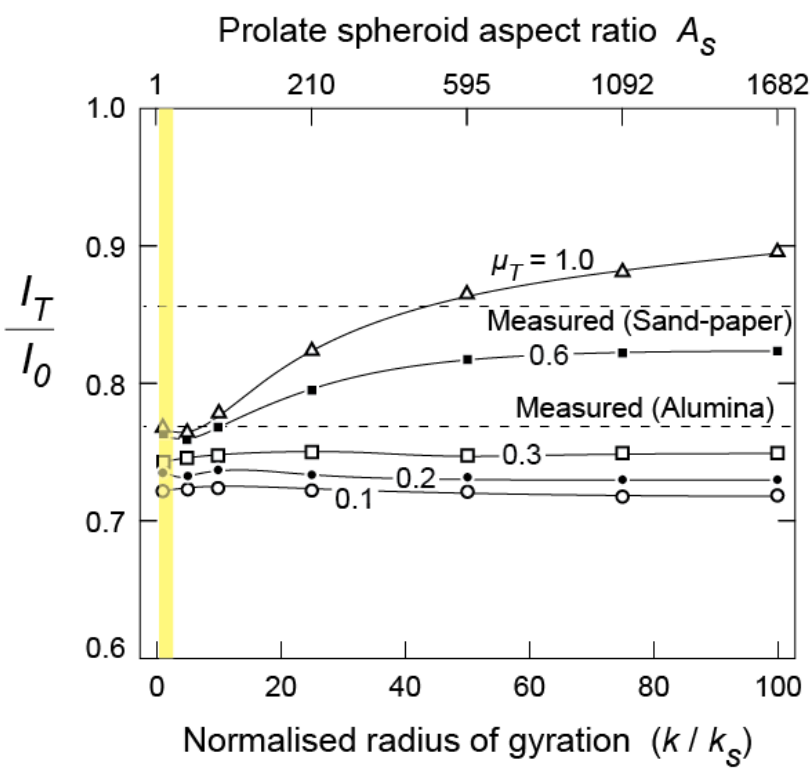

Figure 20: Predictions of the transmitted momenta (a) and (b) as a function of the normalised radius of gyration of the particles for impact of the uniform density sand slug against the inclined target. Calculations are shown for 5 selected values of the particle/target surface friction coefficient. The measured values of the transmitted momenta for impact of the dry sand slug against the inclined target with the Alumina and sand-paper coatings are also indicated. The upper axis indicates the aspect ratio of prolate spheroids with radius of gyration about their minor axis and the realistic range of values for the sand particles used in these experiments is marked by the shaded region.

In order to understand the sensitivity of the transmitted momentum to , we consider the simple situation of the rolling/sliding motion of a single particle along the surface of the target. This particle has a $2^{\text {nd }}$ moment of inertia and diameter (recall the artificial situation we are considering here where even though the particle shape is fixed as a sphere of diameter, is treated as an independent parameter). The particle is given a pure sliding velocity along the surface of the target at time and subjected to a constant normal force. The frictional force acts to reduce the translational velocity 
of the particle while simultaneously increasing its rotational velocity . With initial conditions and at time, the equations of motion of the particle are

and

Sliding terminates with the particle commencing pure rotational motion when . Thereafter, the frictional force reduces to zero as there is no relative sliding between the particle and the target and the particle continues to translate at a steady-state translational velocity and rotational velocity. These are given from Eqs. (6.2) and (6.3) as

The transmitted momentum into the target (in a direction parallel to the surface of the target) is and the normalised transmitted momentum (where is the initial momentum of the particle) then follows as

Thus, for a particle with, while as when rotational motion of the particles is inhibited. This simple analysis illustrates the role that the $2^{\text {nd }}$ moment of inertia plays in governing the momentum that is transmitted into the target in a direction parallel to the target surface due to frictional interactions between the target and the particle. Critically, particles with a large radius of gyration will result in larger transmitted momentum . We thus argue that the discrepancy between the predictions of Section 5 and measurements are due to the fact that the particles were modelled as spherical while in the experiments, the particles have a sub-spherical shape (Fig. 3) that reduces the tendency of the actual sand particles to rotate.

\subsection{Discussion on effect of particle shape}

The measures of transmitted momenta and increase with as seen in Fig. 20. These calculations were performed using spherical particles in which was artificially treated as an independent parameter. In order to gauge the particle shapes corresponding to the range of values covered in Fig. 20 we consider particles that are prolate spheroids with aspect ratio and volume equal to that of a sphere of diameter. Then the radius of gyration of this prolate spheroid about its minor axis is given by the relation

We include a second axis in Fig. 20 (upper scale) to indicate the relation between and given by Eq. (6.6). The values of that bring the measurements and predictions into agreement for the sand-paper coated targets correspond to large values of . Prolate spheroids with such large aspect ratios would have a needle-like appearance 
while the sand particles in the experiments are sub-spherical (Fig. 3). Moreover, we note from Figs. 20a and 20b that there is no single value of that will accurately predict the measured values of both and .

The need for these unrealistically large values of (the realistic range is indicated in Fig. 20) is due to the artificial assumption used in the calculations wherein the particles retain their spherical geometry but is varied independently. For example consider a cubic particle with volume equal to that of a sphere of diameter. Then the radius of gyration of this cubic particle about one of its principal axes is

This cubic particle will never attain a state of pure rolling and for the situation discussed in Section 6.2, will result in a normalised transmitted momentum in a direction parallel to the target surface even with this relatively low value of. We thus argue that in order to predict the transmitted momentum we need to capture the effect of friction between the particles and the target surface accurately. This will require us to appropriately parameterise shapes of the particles used in the experiments and develop discrete element simulations that explicitly model such particles. Experimental verification of this hypothesis using spherical particles would have been ideal. However, spherical particles (usually made of glass) shatter in impact experiments thus making such studies infeasible. A follow-on numerical study [47] wherein particles of different shapes are explicitly modelled verifies the qualitative arguments on the effect particle shape presented here.

\section{Concluding remarks}

The responses of dry and water saturated sand slugs impacting normally oriented and inclined rigid-stationary targets have been measured while simultaneously quantifying the dependence of transmitted momentum on the surface type. Four surface coatings: Alumina, PTFE, Aluminium and sand-paper were employed in this study. Coupled discrete particle/Lagrangian simulations of the experiments are also reported in order to help clarify the physics of the impact process.

The measurements indicate that the slugs deform by spreading along the target surfaces with little rebound of the sand particles. This is reminiscent of the impact of a water jet impacting a rigid target. No measurable differences in the fraction of the transmitted momentum are observed between dry and water saturated sand slugs for both the normal and inclined impact cases. Moreover, over the range of slug velocities investigated here, no effects of slug velocity on the fraction of the transmitted momenta were measured. Symmetry of impact process for the normally oriented target implies that the only non-zero component of the transmitted net momentum is in the direction of the incoming sand slug (i.e., while ) and this also results in no measurable effect of the surface type on the transmitted impulse. By contrast, impact of the sand slug on the target inclined at to the incoming slug results in asymmetric spreading of the sand particles along the surface of the target. This implies that both and and frictional interactions between the sand particles and the target surface now 
play a crucial role in modulating the transmitted momentum. In the absence of friction between the sand particles and the target surface we show that for a target inclined at . However, for all surface coatings employed here, we observed with increasing in the order of surface coatings Alumina to PTFE to Aluminium to sand-paper. Correspondingly, the resultant transmitted momentum also increased in the same order.

Coupled discrete particle/Lagrangian simulations of these experiments using spherical particles predicted the measurements for the normal impact accurately and captured the observed insensitivity of the measurements to slug velocity and surface type as parameterised by the particle/target friction coefficient. However for inclined impact, the simulations considerably under-predicted the measured values of especially in the case of the targets with the sand-paper coated surface. No value of the particle/target friction coefficient brought the measurements and predictions into agreement. We have demonstrated that increasing the radius of gyration of the particles and thereby reducing their tendency to rotate and increases the predicted. With the sand particles employed in the experiments having a sub-spherical we argue that this is the main source of the discrepancy between measurements and predictions.

The combined experimental and numerical study presented here has demonstrated the importance of particle shape in governing the momentum transfer to targets for asymmetric sand slug impacts. Such impacts are more likely to occur in practical situations compared to symmetrical impacts. Most numerical calculations tend to use spherical particles to represent the impacting granular media and neither models that permit rotation of the spherical particles [2] or completely restrict their rotation [5] can accurately capture all components of the transmitted momentum. This study has demonstrated the limitation of the widely used spherical particle assumption in granular impact calculations and indicates the need for more accurate representations of actual particle shapes in numerical calculations.

\section{Acknowledgements}

The work was supported by the Office of Naval Research Grant N00014-09-1-0573 (Program manager, Dr. David Shifler) and the Defense Advanced Projects Agency under grant number W91CRB-11-1-0005 (Program manager, Dr. J. Goldwasser).

\section{References}

[1] Dharmasena KP, Wadley HNG, Liu T, Deshpande VS. The dynamic response of edge clamped plates loaded by spherically expanding sand shells. International Journal of Impact Engineering 2013;62:182-95. doi:10.1016/ j.ijimpeng.2013.06.012. 
[2] Liu T, Fleck NA, Wadley HNG, Deshpande VS. The impact of sand slugs against beams and plates: Coupled discrete particle/finite element simulations. Journal of the Mechanics and Physics of Solids 2013;61:1798-821. doi: 10.1016/j.jmps.2013.03.008.

[3] Rimoli JJ, Talamini B, Wetzel JJ, Dharmasena KP, Radovitzky R, Wadley HNG. Wet-sand impulse loading of metallic plates and corrugated core sandwich panels. International Journal of Impact Engineering 2011;38:837-48. doi:10.1016/j.ijimpeng.2011.05.010.

[4] Wadley HNG, Børvik T, Olovsson L, Wetzel JJ, Dharmasena KP, Hopperstad OS, Deshpande VS, Hutchinson JW. Deformation and fracture of impulsively loaded sandwich panels. Journal of the Mechanics and Physics of Solids 2013;61:674-99. doi:10.1016/j.jmps.2012.07.007.

[5] Borvik T, Olovsson L, Hanssen AG, Dharmasena KP, Hansson H, Wadley HNG. A discrete particle approach to simulate the combined effect of blast and sand impact loading of steel plates. Journal of the Mechanics and Physics of Solids 2011;59:940-58. doi:10.1016/j.jmps.2011.03.004.

[6] Hlady S. Effect of soil parameters on landmine blast. 18th Military Aspects of Blast and Shock (MABS) Conference, Bad Reichenhall, Germany 2004.

[7] Pickering EG, Chung Kim Yuen S, Nurick GN, Haw P. The response of quadrangular plates to buried charges. International Journal of Impact Engineering 2012;49:103-14. doi:10.1016/j.ijimpeng.2012.05.007.

[8] Anderson CE, Behner T, Weiss CE. Mine blast loading experiments. International Journal of Impact Engineering 2011;38:697-706. doi:10.1016/ j.ijimpeng.2011.04.005.

[9] Bergeron D, Tremblay JE. Canadian research to characterize mine blast output. Proceedings of the 16th International MABS Symposium, Oxford, UK 2000.

[10] Follett S, Analysis L, Composite OF, Hulls S, Experimental N, Approach N. Blast analysis of composite V shaped hulls: An experimental and numerical approach. PhD Thesis, Cranfield University, UK 2011.

[11] Fox DM, Huang X, Jung D, Fourney WL, Leiste U, Lee JS. The response of small scale rigid targets to shallow buried explosive detonations. International Journal of Impact Engineering 2011;38:882-91.

[12] Westine PS, Morris BL, Cox PA, Polch E. Development of computer program for floor plate response from land mine explosions. Contract Report No 1345 for US Army TACOM Research and Development Centre 1985.

[13] Morris BL. Analysis of improved crew survivability in light vehicles subjected to mine blast. Final Report for Contract No DAAK70-92-C-0058 for the US Army Belvoir RDEC, Ft Belvoir, VA 1993.

[14] Bergeron D, Walker R, Coffey C. Detonation of 100-gram anti-personnel mine surrogate charges in sand-A test case for computer code validation. Suffield Report No 668, Defence Research Establishment Suffield, Ralston, Alberta, Canada 1998. 
[15] Neuberger A, Peles S, Rittel D. Scaling the response of circular plates subjected to large and close-range spherical explosions. Part II: Buried charges. International Journal of Impact Engineering 2007;34:874-82. doi:10.1016/ j.ijimpeng.2006.04.002.

[16] Reichenbach H, Behrens K, Kuhl AL. Exploratory depth-of-burst experiments. Interim Report, CONTRACT No DNA 001-91-C-0039, Prepared for: Defense Nuclear Agency, Washington, DC, USA 1991. doi:10.1017/ CBO9781107415324.004.

[17] Fairlie G, Bergeron D. Numerical Simulation of Mine Blast Loading on Structures. 17th Military Aspects of Blast Symposium, Las Vegas, Nevada 2002:2-9.

[18] Fourney W, Leiste U, Bonenberger R, Goodings D. Explosive impulse on plates. Fragblast 2005;9:1-17.

[19] Deshpande VS, McMeeking RM, Wadley HNG, Evans A. Constitutive model for predicting dynamic interactions between soil ejecta and structural panels. Journal of the Mechanics and Physics of Solids 2009;57:1139-64. doi:10.1016/ j.jmps.2009.05.001.

[20] Grujicic M, Pandurangan B, Cheeseman BA. The effect of degree of saturation of sand on detonation phenomena associated with shallow-buried and groundlaid mines. Shock and Vibration 2006;13:41-61.

[21] Grujicic M, Pandurangan B, Qiao R, Cheeseman BA, Roy WN, Skaggs RR, Gupta R. Parameterization of the porous-material model for sand with different levels of water saturation. Soil Dynamics and Earthquake Engineering 2008;28:20-35. doi:10.1016/j.soildyn.2007.05.001.

[22] Grujicic M, Pandurangan B, Mocko GM, Hung ST, Cheeseman BA, Roy WN, Skaggs RR. A Combined Multi-Material Euler/Lagrange Computational Analysis of Blast Loading Resulting from Detonation of Buried Landmines. Multidiscipline Modeling in Materials and Structures 2008;4:105-24. doi: $10.1163 / 157361108784050086$.

[23] Wang Z, Hao H, Lu Y. A three-phase soil model for simulating stress wave propagation due to blast loading. International Journal for Numerical and Analytical Methods in Geomechanics 2004;28:33-56. doi:10.1002/nag.325.

[24] Foedinger J. Methodology for improved characterization of landmine explosions, SBIR Phase-II plus program. 2005. Proceedings of the Technical Interchange Meeting, Material Science Corporation 2005.

[25] Holloman RL, Deshpande V, Wadley HNG. Impulse transfer during sand impact with a solid block. International Journal of Impact Engineering 2015;76:98-117. doi:10.1016/j.ijimpeng.2014.09.010.

[26] Holloman RL, Deshpande V, Wadley HNG. Impulse transfer during sand impact with a cellular structure. International Journal of Impact Engineering 2015. doi:10.1016/j.ijimpeng.2015.01.004. 
[27] Park S, Uth T, Fleck NA, Wadley HNG, Deshpande VS. Sand column impact onto a Kolsky pressure bar. International Journal of Impact Engineering 2013;62:229-42. doi:10.1016/j.ijimpeng.2013.07.003.

[28] Benedetti RE. Mitigation of explosive blast effects on vehicle floorboard. Master Thesis, University of Maryland, College Park, MD, USA 2008.

[29] Uth T, Wadley HNG, Deshpande VS. The effect of inclination and stand-off on the dynamic response of beams impacted by slugs of a granular material. International Journal of Solids and Structures 2015;56-57:154-74. doi:10.1016/ j.ijsolstr.2014.11.019.

[30] Cheng X, Varas G, Citron D, Jaeger H, Nagel S. Collective Behavior in a Granular Jet: Emergence of a Liquid with Zero Surface Tension. Physical Review Letters 2007;99:188001. doi:10.1103/PhysRevLett.99.188001.

[31] Johnson CG, Gray JMNT. Granular jets and hydraulic jumps on an inclined plane. Journal of Fluid Mechanics 2011;675:87-116. doi:10.1017/jfm.2011.2.

[32] Uth T, Deshpande VS. Response of clamped sandwich beams subjected to high-velocity impact by sand slugs. International Journal of Impact Engineering 2014;69:165-81. doi:10.1016/j.ijimpeng.2014.02.012.

[33] Liang Y, Spuskanyuk AV, Flores SE, Hayhurst DR, Hutchinson JW, McMeeking RM, Evans AG. The Response of Metallic Sandwich Panels to Water Blast. Journal of Applied Mechanics 2007;74:81. doi: $10.1115 / 1.2178837$.

[34] Tilbrook MT, Deshpande VS, Fleck N a. The impulsive response of sandwich beams: Analytical and numerical investigation of regimes of behaviour. Journal of the Mechanics and Physics of Solids 2006;54:2242-80. doi:10.1016/j.jmps. 2006.07.001.

[35] Pingle SM, Fleck NA, Wadley HNG, Deshpande VS. Discrete element calculations of the impact of a sand column against rigid structures. International Journal of Impact Engineering 2012;45:74-89. doi:10.1016/ j.ijimpeng.2011.10.005.

[36] Plimpton S. Fast parallel algorithms for short range molecular dynamics. Journal of Computational Physics 1995;117:1-19.

[37] Cundall PA, Strack ODL. A discrete numerical model for granular assemblies. Géotechnique 1979;29:47-65. doi:10.1680/geot.1979.29.1.47.

[38] Campbell CS, Brennen CE. Chute flows of granular material: Some computer simulations. Journal of Applied Mechanics 1985;52:172-8.

[39] Campbell CS. Granular shear flows at the elastic limit. Journal of Fluid Mechanics 2002;465:261-91. doi:10.1017/S002211200200109X.

[40] Goel A, Uth T, Liu T, Wadley HNG, Deshpande VS. Coupled discrete/ continuum simulations of the impact of granular slugs with clamped beams: Stand-off effects. Submitted for Publication n.d. 
[41] Liu T, Wadley HNG, Deshpande VS. Dynamic compression of foam supported plates impacted by high velocity soil. International Journal of Impact Engineering 2014;63:88-105. doi:10.1016/j.ijimpeng.2013.08.004.

[42] Bathurst RJ, Rothenburg L. Micromechanical aspects of isotropic granular assemblies with linear contact interactions. Journal of Applied Mechanics 1988;55:17-23.

[43] Silbert L, Ertas D, Grest G, Halsey T, Levine D, Plimpton S. Granular flow down an inclined plane: Bagnold scaling and rheology. Physical Review E 2001;64:051302. doi:10.1103/PhysRevE.64.051302.

[44] Guttenberg N. Microscopic dissipation in a cohesionless granular jet impact. Physical Review E 2012;85:051303. doi:10.1103/PhysRevE.85.051303.

[45] Cho GC, Dodds J, Santamarina JC. Particle Shape Effects on Packing Density, Stiffness , and Strength : Natural and Crushed Sands. Journal of Geotechnical and Geoenvironmental Engineering 2006:591-602.

[46] Farhadi S, Behringer RP. Dynamics of Sheared Ellipses and Circular Disks: Effects of Particle Shape. Physical Review Letters 2014;112:148301. doi: 10.1103/PhysRevLett.112.148301.

[47] Goel A, Wadley HNG, Deshpande VS. Impact of granular slugs on rigid targets: effect of particle shape. Submitted for Publication n.d. 